\title{
Modelagem da gestão de estoques de peças de reposição através de cadeias de Markov
}

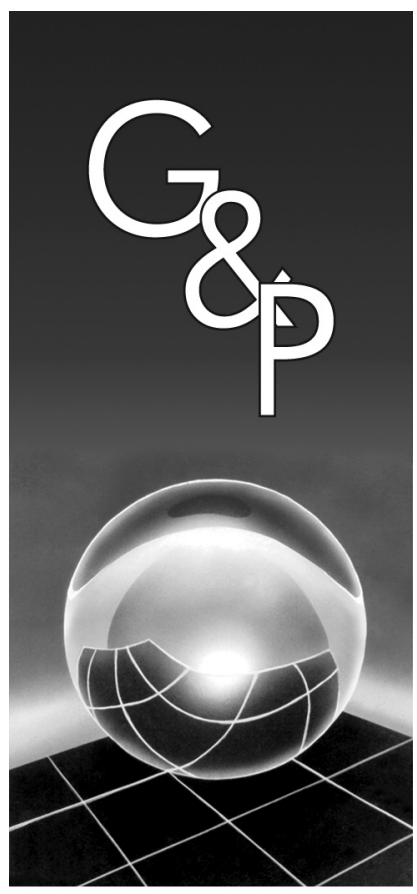

Antonio Vinicius Pimpão Gomes

Peter Wanke

\section{Resumo}

Nessa pesquisa é apresentada uma abordagem para gestão de estoques de peças de reposição com base em cadeias de Markov. É feita uma comparação com a simulação convencional, a fim de validar esta abordagem, bem como é apresentada uma heurística para determinação dos parâmetros da política $(S, s)$ de gestão de estoques, dado um conjunto de itens de custo (falta, excesso e ressuprimento) e de demanda com distribuição Poisson. A análise dos gráficos desses itens de custo em função dos parâmetros da política $(S, s)$ fornece os trade-offs básicos para a formulação da heurística.

Palavras-chave: Peças de reposição. Gestão de estoques. Cadeias de Markov.

\section{Introdução}

Uma típica empresa fabricante de bens de consumo tende a manter entre US\$ 5 a 15 milhões de dólares imobilizados em peças de reposição, a um custo de oportunidade anual que oscila entre 20 a $40 \%$ do valor em estoque (SANDVIG; ALLAIRE, 1998). De modo geral, há um consenso de que os estoques de peças de reposição não podem ser gerenciados pelos modelos ou métodos tradicionais, já que as condições para sua aplicação não são satisfeitas. Isso porque o padrão de consumo é esporádico, ou seja, é irregular e pequeno, os tempos de resposta do ressuprimento são longos e os custos de aquisição são elevados (BOTTER; FORTUIN, 2000). Mesmo assim, as perguntas básicas da gestão de estoques ainda devem ser respondidas, são elas: 'quais itens devem ser estocados' e 'quanto de cada item deve ser mantido em estoque'.

A gestão das peças de reposição também pode ser entendida pelo aspecto do serviço ao cliente, e não apenas pelo aspecto financeiro e/ou de operações. Para muitas empresas que enfrentam um ambiente competitivo mais acirrado, a satisfação dos clientes é crucial (FIGUEIREDO et al., 2003). Um meio bastante comum de manter os clientes satisfeitos é na assistência pós- venda, através do rápido reparo dos produtos e sistemas defeituosos. Para isso, uma quantidade suficiente de peças de reposição deve ser mantida em estoque para atender determinados níveis de serviço ao cliente. O serviço ao cliente pode ser normalmente medido em termos de disponibilidade de produto, como nos indicadores de probabilidade de não faltar produto em estoque e de fill rate (SILVER; PETERSON, 1985; WANKE, 2003), e também em termos de prazo de entrega.

Sob o prisma da administração de materiais, as peças de reposição podem ser divididas em duas principais categorias: itens reparáveis ou itens consumíveis ou descartáveis (BOTTER; FORTUIN, 2000). Os itens reparáveis englobam as peças de reposição que são técnica e economicamente recuperáveis. Em caso de falha, a peça antiga é substituída por uma nova e enviada para um centro de reparo, sendo posteriormente disponibilizada em estoque. Já os itens consumíveis correspondem às peças que não são técnica nem economicamente recuperáveis. Em caso de falha, a peça antiga é simplesmente descartada. No primeiro caso, a possibilidade de recuperação de um item acarreta implicações na gestão de estoques, uma vez que, em linhas gerais, as quantidades em processo de 
recondicionamento devem ser deduzidas das quantidades a serem ressupridas no futuro (SHERBROOKE, 1968; KIM et al., 1996).

Essa pesquisa tem como objetivo principal aplicar os principais conceitos de cadeias de Markov na gestão de estoques de peças de reposição descartáveis. Mais especificamente, deseja-se determinar um procedimento heurístico (não otimizante) de apoio à decisão e escolha dos principais parâmetros da política de estoques $(S, s)$ - nível máximo de estoque e ponto de pedido - a partir do histograma de possíveis estados para a posição final de estoque. Dentre as características da heurística desenvolvida destacam-se: a facilidade de implementação, a consideração dos principais itens ou componentes de custos envolvidos na gestão de estoques de peças de reposição (falta, excesso e ressuprimento) e a proximidade com a solução ótima.

O artigo está estruturado em quatro seções. Na seção 2, é apresentada a problemática da gestão de peças de reposição, bem como as diferentes abordagens encontradas na literatura. Na seção 3, é mostrado como as cadeias de Markov podem auxiliar na gestão de estoque de peças de reposição, principalmente através da geração da distribuição de freqüências (histograma) da posição final de estoque. Finalmente, na seção 4 é apresentada a heurística para determinação do nível máximo de estoque e do ponto de pedido numa política $(S, s)$, sendo que $S$ representa o nível máximo de estoque e $s$ o ponto de pedido. Por fim, são apresentadas as conclusões da pesquisa.

\section{Gestão de estoques de peças de reposição}

A gestão de estoques tem recebido substancial atenção dos meios acadêmico e empresarial nos últimos anos. A maior parte da literatura está focada em determinar, estabelecer ou aplicar métodos para ressuprimento dos estoques em ambientes de produção e distribuição (BOTTER; FORTUIN, 2000). Nesses ambientes, a demanda e o tempo de resposta tendem a ser previstos com maior grau de certeza, e a grande maioria dos modelos ou políticas de estoques empregada permite a tomada de decisões adequadas sobre quanto de cada item ou produto manter em estoque (ver, por exemplo, SILVER et al., 1998). Nesse artigo, a tomada de decisão em gestão de estoques ocorre num contexto totalmente diferente, ou seja, o ressuprimento de peças de reposição. Nesse contexto, empresas e gerentes enfrentam um problema mais complexo: as peças de reposição são caras, a demanda é errática e difícil de prever, os tempos de resposta são geralmente longos, decorrentes de importação, e os clientes querem receber os pedidos rapidamente.
Essa é uma das principais razões para a discussão, sobre qual política de estoques adotar, ficar em segundo plano na literatura sobre peças de reposição. Os principais enfoques tendem a ser: a definição de diferentes objetivos de custo ou serviço, a escolha de premissas mais adequadas sobre a distribuição de probabilidade da demanda e, eventualmente, a escolha do ferramental empregado na modelagem do problema. Nesse contexto, a questão sobre qual política de estoques deve ser utilizada (se, por exemplo, (S, s), (S, S - 1) ou mesmo tamanho de lote fixo Q e ponto de pedido $s$ ) passa a ser um elemento adicional da modelagem. Silver et al. (1998) e Zipkin (2000) fornecem uma descrição bastante detalhada das vantagens e desvantagens dessas políticas para os itens de alto giro (ou classe A de consumo) nos ambientes de produção e de distribuição.

Dessa forma, a literatura sobre o ressuprimento de peças de reposição tende a ser mais escassa (BOTTER; FORTUIN, 2000) e os desenvolvimentos nos últimos anos tendem a apresentar vários possíveis objetivos para o problema, como a determinação do último pedido (HILL et al., 1999), a determinação do intervalo ótimo de revisão (SHIBUYA et al., 1998) e a determinação de políticas de estoque com base na criticidade dos itens (DEKKER et al., 1998).

Além dos diferentes objetivos, não menos variados são as premissas consideradas e os contextos de tomada de decisão verificados nas diferentes pesquisas. Por exemplo, Wanke (2005) aproxima a demanda pela distribuição Gama, e a partir do confronto entre dados reais e previstos de consumo, determina os impactos do erro de previsão nos níveis médio de estoque e de disponibilidade do item (fill rate).

Já Walker (1997) modela a gestão de estoques de peças de reposição através da política $(\mathrm{S}, \mathrm{S}-1)$, tão logo o nível de estoque baixe de uma unidade, é solicitado um ressuprimento para retornar ao nível máximo de estoque. É assumido que a taxa de falha de uma peça segue uma distribuição exponencial. Com base na probabilidade de uma dada quantidade de falhas (estado estacionário), o autor oferece meios para se determinar graficamente a quantidade de peças que se deve manter em estoque em função da quantidade de máquinas e da relação entre o tempo de reparo e o tempo médio de operação.

Contudo, a utilização de cadeias de Markov no tratamento de questões relacionadas à manutenção e à gestão de peças de reposição tem se tornado recorrente na literatura. Por exemplo, Smidt-Destombes et al. (2006) propõem um modelo para a política de manutenção de uma fábrica que funciona com $N$ máquinas que dependem de uma peça para funcionar, onde todas têm a mesma probabilidade de falha. A partir deste modelo, visa-se analisar o processo, comparando-se o comportamento do nível de peças em estoque, da política de manutenção e da 
prioridade de reparo com o nível de serviço do sistema, mensurado a partir da razão entre o tempo de reparo e o tempo total da operação.

Wong et al. (2005) desenvolveram um método analítico para avaliar o desempenho de um sistema de estoques compartilhado por várias companhias, através da estimação de indicadores de desempenho, como fill rate e o tempo ocioso devido à falta. A estratégia de compartilhamento de estoques por diferentes companhias do mesmo segmento de mercado, segundo os autores, constitui uma maneira de aperfeiçoar o desempenho logístico da empresa, sem incorrer em custos adicionais. A modelagem baseia-se em cadeias de Markov, a partir das quais são estimados esses indicadores de desempenho.

Sleptchenko et al. (2005) modelaram um modelo de filas com $n$ servidores e $k$ classes para analisar a área de consertos de peças de reposição numa empresa. Seu objetivo era determinar como o estabelecimento de regras de prioridades para o conserto poderia afetar os custos totais de operação. Finalmente, Vaughan (2005) propõe uma política $(\mathrm{S}, \mathrm{s})$ de gestão de estoques de peças de reposição obtida através de um modelo de programação estocástica dinâmica. Ambas as modelagens foram por cadeias de Markov.

Conforme mencionado, o principal objetivo dessa pesquisa é aplicar o conceito de cadeias de Markov na gestão de peças de reposição. Para tanto, será discutido nas próximas seções como obter o histograma de possíveis estados para a posição final (estacionária) de estoque. Esse histograma, ao permitir o cálculo dos principais componentes de custos envolvidos na gestão de estoques de peças de reposição (falta, excesso e ressuprimento) constitui a base para determinação de um procedimento heurístico, isto é, não necessariamente otimizante e de apoio à decisão e escolha dos principais parâmetros de uma política de estoques (S, s) - nível máximo de estoque e ponto de pedido.

\section{Aplicando cadeias de Markov à gestão de estoques de peças de reposição}

As cadeias de Markov são um caso particular de processo estocástico com tempo discreto. Apresentam a propriedade de que os estados anteriores são irrelevantes para a predição dos estados seguintes, desde que o estado atual seja conhecido. Possuem aplicação em diversas áreas da engenharia e seu interesse em operações, sobretudo na área de gestão de estoques, é crescente, conforme apontado na seção anterior.

\subsection{Determinação do vetor de estados e da matriz de transição de estados}

Em Hillier e Lieberman (1988), tem-se um exemplo clássico de utilização de cadeias de Markov para a gestão de estoques. Nesses autores, foram definidas as transições entre os estados possíveis para a posição de estoque de um dado item e sua probabilidade estacionária. Nessa pesquisa, o escopo do problema é ampliado pela especificação do estado da falta de estoque (estoque zero ou negativo) e pela possibilidade do estado variar em função do ponto de pedido, além do nível máximo de estoque. Esse escopo ampliado implicou na determinação de uma heurística para o apoio à tomada de decisão quanto aos parâmetros mais adequados da política de estoques (nível máximo de estoque e ponto de pedido), em função de diferentes condições da demanda e dos custos da falta, do excesso e de ressuprimento.

Numa cadeia de Markov aplicada à gestão de estoques, os estados possíveis denotam as diferentes posições de estoques que podem ocorrer ao longo do tempo (HILLIER; LIEBERMAN, 1988). Assim, por exemplo, caso o nível máximo estoque $(S)$ de uma determinada peça seja de 20 unidades, a cadeia de Markov terá 22 estados possíveis. São eles: de backorders (ou pendências), caracterizado por estoques negativos, e as sucessivas posições de estoque que vão de zero até vinte unidades. Em linhas gerais, para uma quantidade máxima $n$ de unidades de uma dada peça em estoque, a cadeia de Markov correspondente terá $n+2$ posições de estoque. Então, o vetor de posições de estoque, que contém a distribuição de probabilidades da quantidade de peças em estoque num determinado instante de tempo $t$, é representado na Figura 1.

Por definição, a soma das probabilidades de todos os estados possíveis deverá ser $1(100 \%)$. Além disso, nenhuma dessas probabilidades poderá ser zero. Assim, $o$ vetor estado referente ao tempo $t$ pode ser interpretado como sendo a distribuição de probabilidade da posição de estoque nesse instante de tempo.

Com relação à matriz de transição, a mesma é definida através das probabilidades de transição entre os estados (HILLIER; LIEBERMAN, 1988). Tais probabilidades são calculadas diretamente a partir das probabilidades de cada estado e também dependem da política de ressuprimento. Dessa forma, a política de estoques escolhida

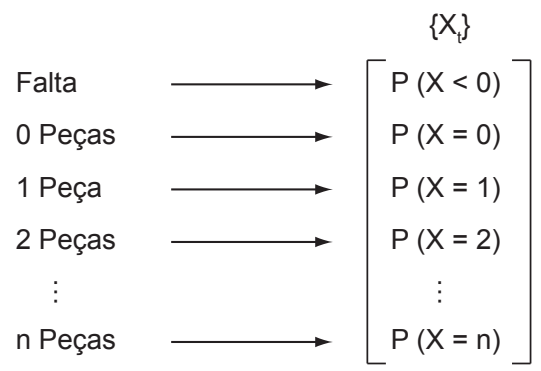

Figura 1. Vetor de estados (posições possíveis de estoque). 
para as peças de reposição tem que estar refletida nas probabilidades da matriz de transição.

Mais especificamente, a política de estoques $(S, s)$ modelada nesse artigo é operacionalizada da seguinte forma: toda vez que a posição de estoque baixar a um nível menor que $s$ (ponto de pedido), deve ser pedido um lote de ressuprimento de tamanho tal que a posição de estoque retorne a um nível $S$ (nível máximo de estoque). É considerado inicialmente, para efeito de simplificação, que o lead-time (ou tempo de resposta) decorrido desde a colocação do pedido até seu recebimento equivale a um único período de tempo. Em outras palavras, que é feito um pedido ao final de um período e que o mesmo é entregue somente ao final do período seguinte. Nessa pesquisa também não são considerados backorders (ou pedidos pendentes), implicando casos em que uma multa ou penalidade é aplicada imediatamente após o momento da falta. Tal prática é bastante comum entre fornecedores de equipamentos e peças de informática para o setor financeiro (mainframes para processamento de dados) e de telecomunicações.

Sejam os termos:

$I P_{t}=$ posição de estoque ao final do período $\mathrm{t}$

$D_{t}=$ demanda ocorrida no tempo $\mathrm{t}$

$Q=$ lote de ressuprimento

É definida a posição de estoque como se segue (ZIPKIN, 2000):

$$
\begin{array}{cc}
\mathrm{IP}_{\mathrm{t}}=\mathrm{IP}_{\mathrm{t}-1}-\mathrm{D}_{\mathrm{t}} \quad \text { se IP } \mathrm{P}_{\mathrm{t}-1}>\mathrm{s} \\
\mathrm{IP}_{\mathrm{t}}=\mathrm{IP}_{\mathrm{t}-1}-\mathrm{D}_{\mathrm{t}}+\mathrm{Q} & \operatorname{se} \mathrm{IP}_{\mathrm{t}-1} \leq \mathrm{s}
\end{array}
$$

Como $Q+I P_{t-1}$ sempre será igual a $S$, ou seja, o nível máximo da posição de estoque, tem-se que:

$$
\begin{array}{ll}
\mathrm{IP}_{\mathrm{t}}=\mathrm{S}-\mathrm{D}_{\mathrm{t}} & \text { se IP } \mathrm{t}_{\mathrm{t}} \leq \mathrm{s} \\
\mathrm{IP}_{\mathrm{t}}=\mathrm{IP}_{\mathrm{t}-1}-\mathrm{D}_{\mathrm{t}} & \text { se IP } \mathrm{It}_{\mathrm{t}-1}>\mathrm{s}
\end{array}
$$

As transições de estados podem ser organizadas na matriz de transição genérica apresentada na Figura 2. Os valores da matriz representam a probabilidade de tran- sição de uma dada posição no tempo $t$ para outra posição no tempo $\mathrm{t}+1$.

Percebe-se que a transição entre os estados depende diretamente da demanda e da política de estoques. A probabilidade de transição de um estado $X$ para um estado $Y$ será igual à probabilidade da demanda ser igual a $\mathrm{X}-\mathrm{Y}$, caso $\mathrm{X}$ seja maior que $s$. Por outro lado, caso $X$ seja menor que $s$, a probabilidade de transição será igual à probabilidade da demanda no período ser igual a $\mathrm{S}-\mathrm{Y}$. Vale notar também, que caso tenhamos $\mathrm{X}>\mathrm{Y}$ e $\mathrm{X}>\mathrm{s}$, a probabilidade de haver uma passagem do estado $X$ para o estado Y será zero, uma vez que tal fato implicaria na ocorrência de uma demanda negativa.

\subsection{Convergência para o estado estacionário}

Uma vez definidos o estado inicial do modelo e a matriz de transição, pode-se inferir a evolução das posições de estoque ao longo do tempo e sua convergência para uma distribuição de freqüências estacionária. Essa distribuição de frequiências da posição de estoques é fundamental para a tomada de decisões relativas à gestão de estoques. Basicamente porque essa distribuição representa um histograma esperado do comportamento do nível de estoque no longo prazo, a partir do qual podem ser confrontados os efeitos, em termos de custo e nível de serviço, de decisões quanto aos pontos de pedido e níveis máximos de estoque.

A convergência para esse estado estacionário é consequiência direta da minimização dos efeitos do nível de estoque inicial com o passar do tempo, o que é intuitivo. O fato de haver, no início do processo, uma elevada quantidade de peças em estoque, em nada afetará a distribuição de probabilidade da posição de estoque após um longo tempo de operação do sistema. Isso é assegurado pelo fato da matriz apresentada na Figura 2 se caracterizar como recorrente positiva e irredutível (GAMERMAN, 1996).

\subsection{Determinação da distribuição estacionária de freqüências}

O cálculo das frequiências dos estados estacionários é feito por sucessivas multiplicações da matriz de transição

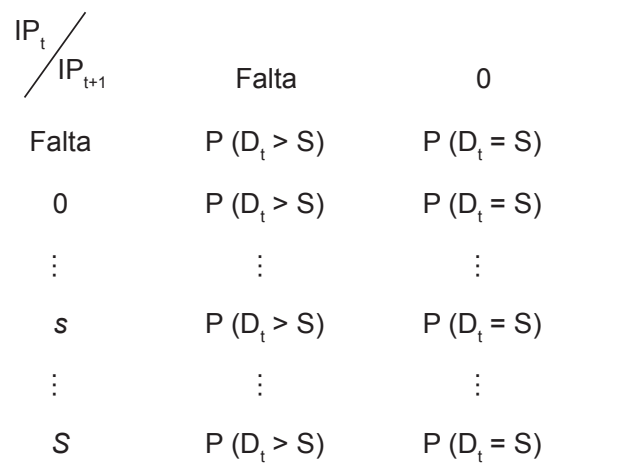

Figura 2. Matriz de transição de estados (posições possíveis de estoque).

$\begin{array}{ccc}s & \ldots & s \\ P\left(D_{t}=s-s\right) & \ldots & P\left(D_{t}=0\right) \\ P\left(D_{t}=s-s\right) & \ldots & P\left(D_{t}=0\right) \\ \vdots & \ldots & \vdots \\ P\left(D_{t}=s-s\right) & \ldots & P\left(D_{t}=0\right) \\ \vdots & \ldots & \vdots \\ P\left(D_{t}=s-s\right) & \cdots & P\left(D_{t}=0\right)\end{array}$


pela respectiva matriz de estado do tempo anterior. Esses passos, em perspectiva, permitem representar a evolução dos níveis de estoque ao longo do tempo (HILLIER; LIEBERMAN, 1988).

Essa multiplicação é relativamente simples de ser efetuada em planilha eletrônica, uma vez que o segundo termo desse produto é uma matriz coluna (Figura 1). Conforme mencionado, essas sucessivas multiplicações convergem atingindo no limite a chamada distribuição estacionária para a posição de estoque. De posse desta informação, têm-se as frequiências esperadas para cada posição de estoque no tempo. Na Tabela 1 é apresentado um exemplo de como a distribuição de freqüências (ou de probabilidades) da posição de estoque converge após um número reduzido de interações. Foi considerada uma política $(S, s)$ com nível máximo de estoque de três unidades e ponto de pedido igual a zero. A demanda obedece à distribuição de Poisson, com média de duas unidades por período.

A partir do sétimo período, as probabilidades para cada estado de estoque tornam-se estacionárias, isto é, não mais variam ao longo do tempo. Dentre as vantagens de se utilizar cadeias de Markov na gestão de estoques de peças de reposição, destaca-se o fato de não ser necessária a obtenção de grandes séries históricas, muitas vezes não confiáveis em função dos aspectos expostos nas seções iniciais desse artigo. Outra vantagem é que a partir da distribuição de freqüências estacionárias podem-se estimar diretamente indicadores como o nível de estoque médio, a freqüência de faltas e a freqüência de ressuprimento.

\subsection{Equivalência com a simulação computacional}

Para demonstrar que a modelagem por cadeias de Markov fornece as mesmas freqüências de posição de estoque que a simulação computacional, para um grande número de corridas (WANKE, 2003 p. 167), foi testada uma mesma política de estoques (S,s) nessas duas abordagens, sendo ao final comparados os histogramas das posições de estoque fornecidos por ambas as abordagens

Tabela 1. Convergência da distribuição de probabilidades da posição de estoque.

\begin{tabular}{cccccc}
\hline Tempo & Falta & $\mathbf{I P}=\mathbf{0}$ & $\mathbf{I P}=\mathbf{1}$ & $\mathbf{I P}=\mathbf{2}$ & $\mathbf{I P}=\mathbf{3}$ \\
\hline 0 & 0,1428 & 0,1804 & 0,2706 & 0,2706 & 0,1353 \\
1 & 0,3138 & 0,2292 & 0,2340 & 0,1607 & 0,0620 \\
2 & 0,2774 & 0,2160 & 0,2389 & 0,1855 & 0,0819 \\
3 & 0,2841 & 0,2187 & 0,2383 & 0,1808 & 0,0778 \\
4 & 0,2830 & 0,2182 & 0,2384 & 0,1816 & 0,0786 \\
5 & 0,2832 & 0,2183 & 0,2384 & 0,1815 & 0,0784 \\
6 & 0,2831 & 0,2183 & 0,2384 & 0,1815 & 0,0785 \\
7 & 0,2831 & 0,2183 & 0,2384 & 0,1815 & 0,0784 \\
\hline
\end{tabular}

(Figura 3). O critério de comparação foi o Teste QuiQuadrado para aderência dessas distribuições (Tabela 2).

Tanto via simulação computacional quanto via cadeias de Markov, testou-se um nível máximo de nove unidades em estoque e um ponto de pedido de seis unidades, com demanda média de duas unidades por período de tempo, obedecendo à distribuição de Poisson. Os resultados sugerem que ambas as distribuições se equivalem. Entretanto, os ganhos em relação a um menor esforço computacional e tempo de execução são substanciais na modelagem por cadeias de Markov. Por exemplo, na simulação feita em planilha Excel foram gerados 10000 valores de demanda e realizadas 50 replicações, a partir das quais foram calculadas as médias e as distribuições para as posições de estoque. Os mesmos resultados em planilha Excel foram obtidos em menos de um minuto

Tabela 2. Comparação dos resultados entre simulação e cadeias de Markov.

\begin{tabular}{cccc}
\hline Estados & $\begin{array}{c}\text { Simulação } \\
\text { computacional }\end{array}$ & $\begin{array}{c}\text { Cadeias de } \\
\text { Markov }\end{array}$ & Teste de Pearson \\
\hline Falta & 3 & 3 & 0,03 \\
0 & 10 & 11 & 0,07 \\
1 & 28 & 40 & 5,06 \\
2 & 141 & 128 & 1,23 \\
3 & 362 & 350 & 0,37 \\
4 & 793 & 803 & 0,13 \\
5 & 1574 & 1496 & 3,84 \\
6 & 2144 & 2183 & 0,72 \\
7 & 2402 & 2384 & 0,13 \\
8 & 1769 & 1816 & 1,23 \\
9 & 775 & 785 & 0,13 \\
\hline Qui-quadrado calculado & \multicolumn{3}{c}{12,97} \\
Qui-quadrado para alfa $=0,01$ & \multicolumn{3}{|c}{23,21} \\
\hline
\end{tabular}

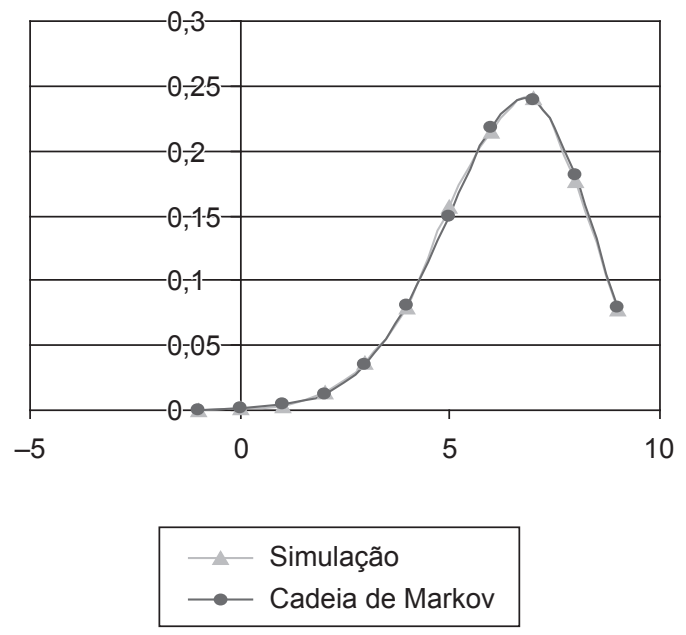

Figura 3. Comparação de histogramas. 


\subsection{Determinação dos custos de estoque a partir do estado estacionário}

Conforme mencionado, a partir da distribuição de frequiências estacionárias podem-se estimar diretamente indicadores como o nível de estoque médio, a freqüência de faltas e a frequiência de ressuprimento. Com base nesses indicadores é possível calcular os principais itens de custos relativos a uma política de estoque: o custo do excesso, o custo da falta e o custo de ressuprimento. Tais custos são os mais utilizados na literatura da gestão de estoques (WANKE, 2003), e a partir deles será desenvolvido o procedimento heurístico que é descrito e testado na próxima seção.

Especificamente, o custo da falta será assumido nesse artigo como uma multa ou penalidade, aplicada quando há falta do item em estoque. Tal penalidade pode não apenas ser reflexo de aspectos contratuais de nível de serviço, mas também de lucros ou receitas cessantes. Por sua vez, o custo do excesso pode ser interpretado como o custo de oportunidade para se manter uma unidade de determinada peça ou item em estoque, podendo ser expresso em termos do valor imobilizado em estoque daquela peça aplicado a uma taxa de oportunidade i. Com relação ao custo de ressuprimento, o mesmo pode ser expresso como sendo o custo de colocação de pedido, geralmente considerado como fixo, ou seja, independente da quantidade pedida.

Portanto, partindo da distribuição estacionária de freqüências, os custos da falta, do excesso e de ressuprimento são estimados conforme discussão a seguir.

\subsubsection{Custo do excesso}

$\mathrm{O}$ custo do excesso segue a fórmula geralmente adotada para o custo de manutenção e carregamento de estoque, dada por:

Custo do excesso $=\mathrm{IP}_{\text {medio }} \cdot \mathrm{Caq} \cdot \mathrm{i}$

em que Caq $=$ custo de aquisição da peça e $\mathrm{i}=$ taxa de oportunidade do capital.

A posição de estoque média é dada por:

$$
\mathrm{IP}_{\text {medio }}=\sum_{\mathrm{j}=0}^{\mathrm{s}} \mathrm{p}_{\mathrm{j}} \cdot \mathrm{j}
$$

em que $\mathrm{j}=$ posição de estoque e $\mathrm{p}_{\mathrm{j}}=$ probabilidade associada à posição de estoque $\mathrm{j}$.

\subsubsection{Custo do ressuprimento}

Já o custo de ressuprimento, ou de pedido, pode ser entendido como o custo fixo associado ao transporte das quantidades pedidas aos fornecedores. Sua probabilidade de ocorrência depende da posição de estoque se situar abaixo do ponto de pedido.

$$
\text { Custo do ressuprimento }=\mathrm{CR} \cdot \sum_{\mathrm{j}=0}^{s-1} \mathrm{p}_{\mathrm{j}}
$$

em que $\mathrm{CR}$ = custo de ressuprimento, incorrido a cada pedido.

\subsubsection{Custo da falta}

Finalmente, o custo da falta é a penalidade decorrente por falta da peça em estoque. Sua probabilidade de ocorrência depende da posição de estoque ser zero.

Custo da falta $=\mathrm{M} \cdot \mathrm{p}_{0}$

em que $\mathrm{M}=$ multa ou penalidade derivada da falta do produto em estoque e $\mathrm{p}_{0}=$ probabilidade da posição de estoque ser zero.

\section{Heurística para determinação dos parâmetros da política $(S, s)$}

Tendo sido abordado como se obter o histograma de possíveis estados para a posição final ou estacionária de estoque, o próximo passo é o desenvolvimento da heurística para determinar os parâmetros da política $(\mathrm{S}, \mathrm{s})$ - nível máximo de estoque e ponto de pedido - bastante utilizada na prática para peças de reposição (VAUGHAN, 2005). Esse histograma, ao permitir o cálculo dos principais componentes de custos envolvidos na gestão de estoques de peças de reposição (falta, excesso e ressuprimento), constitui a base para determinação do procedimento heurístico (não otimizante) de apoio à decisão e escolha dos principais parâmetros dessa política. Deve ser lembrado que, além dos componentes de custo apresentados, a demanda segue uma distribuição Poisson.

Com relação ao entendimento do impacto desses parâmetros nos três componentes de custo, os gráficos apresentados na Figura 4 ilustram o comportamento típico da probabilidade de falta, da probabilidade de ressuprimento e do estoque médio, para cada dupla ordenada $(\mathrm{S}, \mathrm{s})$. As características dessas curvas em relação ao nível ou limite máximo de estoque e ao ponto de pedido oferecem um ponto de partida bastante útil para a generalização de procedimentos em busca de parâmetros ótimos ou próximos do ótimo.

Por exemplo, com relação à probabilidade de falta, nota-se que ela apresenta uma queda muito acentuada em relação ao aumento do ponto de pedido e ao aumento do nível ou limite máximo de peças em estoque. Tal fato tem relação lógica e direta com o aumento do estoque médio, o que pode ser conseguido pelo incremento de apenas um ou ambos os parâmetros $\mathrm{S}$ e s.

Entretanto, aumentos em S e s acarretam efeitos diferentes na probabilidade ou freqüência de ressuprimento. Por exemplo, dado um ponto de pedido fixo, o aumento do limite máximo adicionará mais estados que o sistema pode assumir acima do ponto de pedido, diminuindo a freqüência de ressuprimento e, conseqüentemente, seu custo. Já o aumento do ponto de pedido, dado um limite 
Ponto de pedido $=0$

Estoque médio vs. limite de estoque

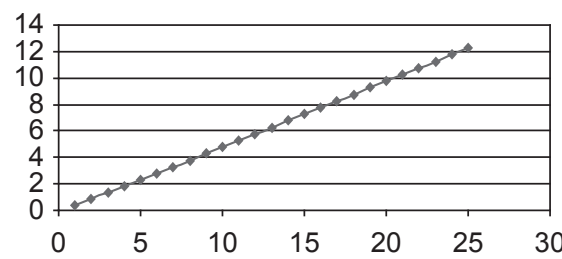

Probabilidade de falta vs. limite de estoque

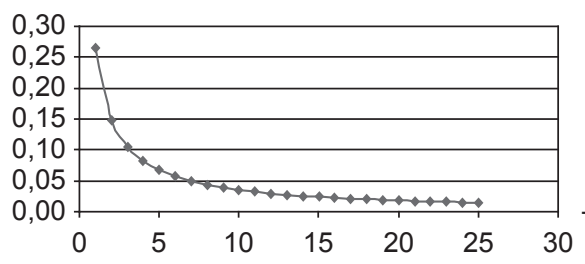

Probabilidade de ressuprimento vs. limite de estoque

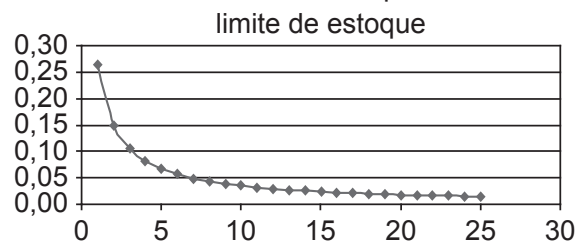

Nível $=25$

Estoque médio vs. ponto de pedido

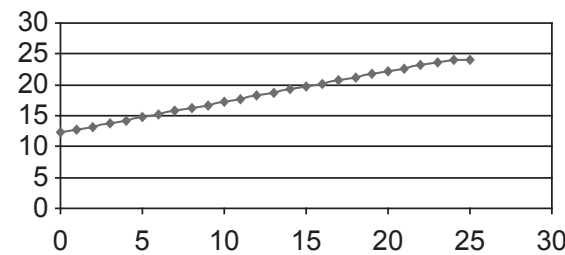

Probabilidade de falta vs. ponto de pedido

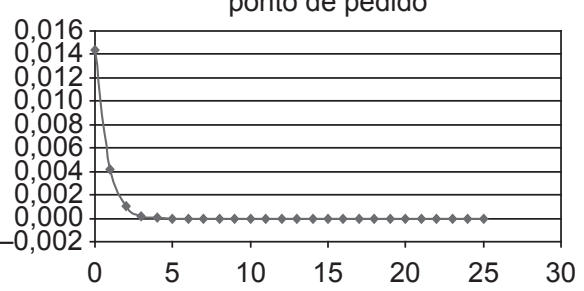

Probabilidade de ressuprimento vs. ponto de pedido

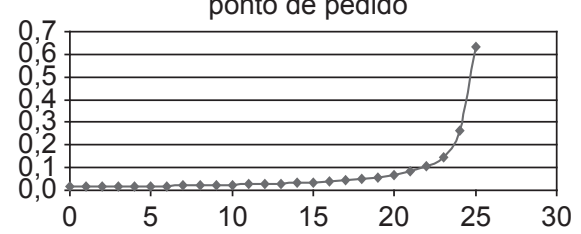

Nível $=10$

Estoque médio vs. ponto de pedido

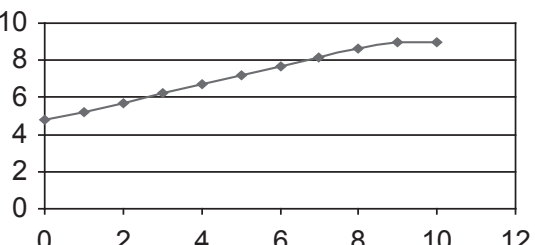

Probabilidade de falta vs. ponto de pedido

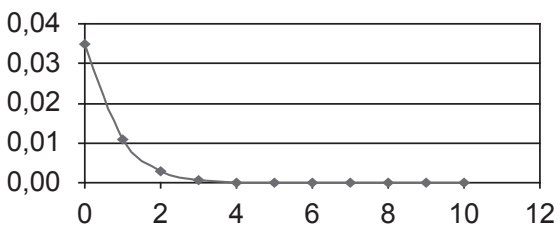

Probabilidade de ressuprimento vs. ponto de pedido

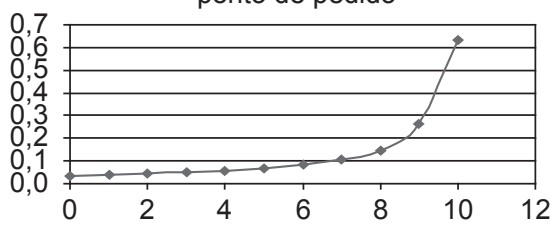

Figura 4. Impactos do nível máximo de estoque e do ponto de pedido.

máximo fixo, aumentará o número de estados possíveis abaixo do ponto de pedido, implicando maior frequiência ou probabilidade de reposição. Em outras palavras, a variação dos custos em relação aos parâmetros da política de estoques é sempre monotônica, sendo que a variação dos custos entre cada parâmetro só difere com relação ao custo de ressuprimento: para o nível ou limite máximo de estoque a variação é negativa, e para o ponto de pedido, positiva.

Em seguida, na Figura 5 e no Quadro 1 é apresentada a heurística proposta para a determinação dos parâmetros da política $(S, s)$. Tal heurística aproxima-se bastante do custo ótimo. Consiste inicialmente na minimização do custo através do nível ou do limite máximo, e a partir da obtenção deste, na minimização do custo em função do ponto de pedido. Este método baseia-se em aproximar a política ótima $\left(\mathrm{S}_{\text {ótimo }}, \mathrm{S}_{\text {ótimo }}\right)$ pela política $(\mathrm{S}, \mathrm{S}-1)$, ou seja, tão logo haja o consumo de uma unidade, coloca-se um pedido de ressuprimento. Uma premissa implícita nessa heurística é o elevado valor do custo da falta em relação aos demais custos, bastante adequada para o caso de peças de reposição, que tendem a ser extremamente críticas.

Quando ocorre a obtenção do custo ótimo em função dos parâmetros $\mathrm{S}$ e s, o fato da demanda ser baixa e, conseqüentemente, do número máximo de posições de estoques ser pequeno, garante um tempo de computação baixo. Ou seja, mesmo testando exaustivamente todas as
Quadro 1. Descrição passo a passo da heurística proposta.

Limite máximo $=0 \mathrm{e}$

Ponto de pedido = limite máximo.

Assumir auxiliar com um valor inicial muito alto.

Custo $=$ custo total (limite máximo, ponto de pedido $)-$ custo

total (limite máximo +1 , ponto de pedido).

Se custo $>0$

então auxiliar $=$ custo, limite superior $=$ limite superior +1 ,

ponto de pedido = limite superior;

senão pare.

Limite ótimo = limite máximo e

Ponto de pedido ótimo = limite ótimo.

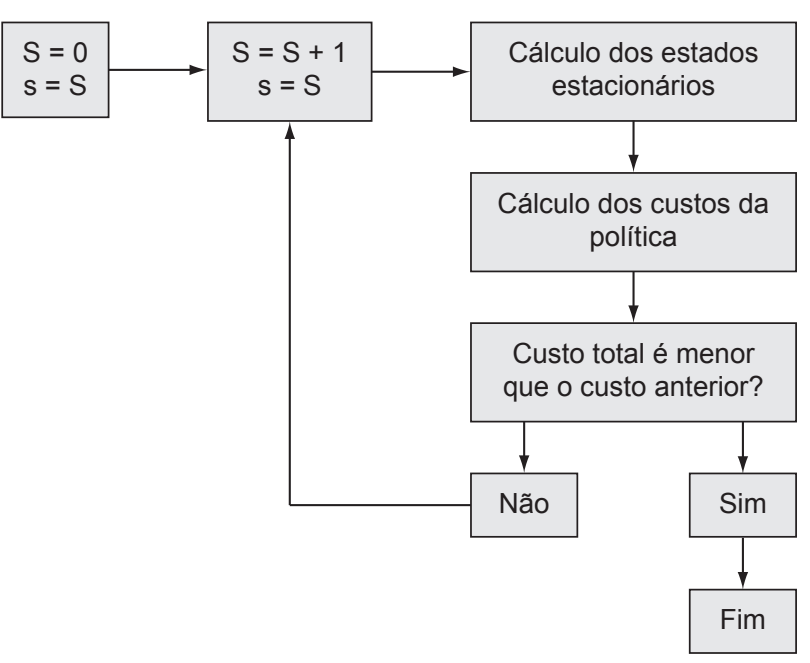

Figura 5. A heurística na representação gráfica de um algoritmo. 
possíveis combinações para o ponto de pedido e para o nível ou limite máximo, o esforço computacional será inferior ao da simulação (conforme seção 3.4). Para mais posições de estoque máximo, o tempo de computação pode aumentar substancialmente. A solução ótima e a solução dada pela heurística são comparadas, caso a caso, nas Tabelas 3 e 4.
Para a construção dos resultados apresentados nas Tabelas 3 e 4, foi assumida uma variação nos custos de aquisição (Caq), além de uma variação na multa incorrida por indisponibilidade da peça $(M)$, de forma a explorar diferentes cenários de custos. O custo de ressuprimento foi mantido constante, igual a 800 unidades monetárias,

Tabela 3. Soluções da heurística.

\begin{tabular}{|c|c|c|c|c|c|c|c|c|}
\hline \multirow[t]{2}{*}{ M } & \multirow[t]{2}{*}{ Caq } & \multicolumn{7}{|c|}{ Média da demanda } \\
\hline & & 0,5 & 1 & 2 & 4 & 6 & 8 & 10 \\
\hline \multirow[t]{5}{*}{250000} & 10000 & $(3,2)$ & $(5,4)$ & $(7,6)$ & $(10,9)$ & $(14,13)$ & $(17,16)$ & $(19,18)$ \\
\hline & 20000 & $(3,2)$ & $(4,3)$ & $(6,5)$ & $(10,9)$ & $(13,12)$ & $(16,15)$ & $(18,17)$ \\
\hline & 30000 & $(3,2)$ & $(4,3)$ & $(6,5)$ & $(9,8)$ & $(12,11)$ & $(15,14)$ & $(18,17)$ \\
\hline & 40000 & $(3,2)$ & $(4,3)$ & $(6,5)$ & $(9,8)$ & $(12,11)$ & $(15,14)$ & $(17,16)$ \\
\hline & 50000 & $(3,2)$ & $(4,3)$ & $(6,5)$ & $(9,8)$ & $(12,11)$ & $(14,13)$ & $(17,16)$ \\
\hline \multirow[t]{5}{*}{500000} & 10000 & $(4,3)$ & $(5,4)$ & $(7,6)$ & $(11,10)$ & $(14,13)$ & $(17,16)$ & $(20,19)$ \\
\hline & 20000 & $(3,2)$ & $(5,4)$ & $(7,6)$ & $(10,9)$ & $(14,13)$ & $(17,16)$ & $(19,18)$ \\
\hline & 30000 & $(3,2)$ & $(5,4)$ & $(7,6)$ & $(10,9)$ & $(13,12)$ & $(16,15)$ & $(19,18)$ \\
\hline & 40000 & $(3,2)$ & $(4,3)$ & $(6,5)$ & $(10,9)$ & $(13,12)$ & $(16,15)$ & $(18,17)$ \\
\hline & 50000 & $(3,2)$ & $(4,3)$ & $(6,5)$ & $(10,9)$ & $(13,12)$ & $(15,14)$ & $(18,17)$ \\
\hline \multirow[t]{5}{*}{750000} & 10000 & $(4,3)$ & $(5,4)$ & $(8,7)$ & $(11,10)$ & $(15,14)$ & $(18,17)$ & $(21,20)$ \\
\hline & 20000 & $(4,3)$ & $(5,4)$ & $(7,6)$ & $(11,10)$ & $(14,13)$ & $(17,16)$ & $(20,19)$ \\
\hline & 30000 & $(3,2)$ & $(5,4)$ & $(7,6)$ & $(10,9)$ & $(14,13)$ & $(17,16)$ & $(19,18)$ \\
\hline & 40000 & $(3,2)$ & $(5,4)$ & $(7,6)$ & $(10,9)$ & $(13,12)$ & $(16,15)$ & $(19,18)$ \\
\hline & 50000 & $(3,2)$ & $(4,3)$ & $(7,6)$ & $(10,9)$ & $(13,12)$ & $(16,15)$ & $(19,18)$ \\
\hline \multirow[t]{5}{*}{1000000} & 10000 & $(4,3)$ & $(6,5)$ & $(8,7)$ & $(12,11)$ & $(15,14)$ & $(18,17)$ & $(21,20)$ \\
\hline & 20000 & $(4,3)$ & $(5,4)$ & $(7,6)$ & $(11,10)$ & $(14,13)$ & $(17,16)$ & $(20,19)$ \\
\hline & 30000 & $(4,3)$ & $(5,4)$ & $(7,6)$ & $(11,10)$ & $(14,13)$ & $(17,16)$ & $(20,19)$ \\
\hline & 40000 & $(3,2)$ & $(5,4)$ & $(7,6)$ & $(10,9)$ & $(14,13)$ & $(17,16)$ & $(19,18)$ \\
\hline & 50000 & $(3,2)$ & $(5,4)$ & $(7,6)$ & $(10,9)$ & $(13,12)$ & $(16,15)$ & $(19,18)$ \\
\hline
\end{tabular}

Tabela 4. Soluções ótimas.

\begin{tabular}{|c|c|c|c|c|c|c|c|c|}
\hline \multirow[t]{2}{*}{$\mathbf{M}$} & \multirow[t]{2}{*}{ Caq } & \multicolumn{7}{|c|}{ Média da demanda } \\
\hline & & 0,5 & 1 & 2 & 4 & 6 & 8 & 10 \\
\hline \multirow[t]{5}{*}{250000} & 10000 & $(3,2)$ & $(5,4)$ & $(7,5)$ & $(11,8)$ & $(14,11)$ & $(17,14)$ & $(19,16)$ \\
\hline & 20000 & $(3,2)$ & $(4,3)$ & $(7,5)$ & $(10,8)$ & $(13,11)$ & $(16,13)$ & $(18,16)$ \\
\hline & 30000 & $(3,2)$ & $(4,3)$ & $(6,5)$ & $(9,8)$ & $(12,10)$ & $(15,13)$ & $(18,15)$ \\
\hline & 40000 & $(3,2)$ & $(4,3)$ & $(6,5)$ & $(9,7)$ & $(12,10)$ & $(15,13)$ & $(17,15)$ \\
\hline & 50000 & $(3,2)$ & $(4,3)$ & $(6,4)$ & $(9,7)$ & $(12,10)$ & $(14,12)$ & $(17,15)$ \\
\hline \multirow[t]{5}{*}{500000} & 10000 & $(4,3)$ & $(5,4)$ & $(8,6)$ & $(11,9)$ & $(14,12)$ & $(17,15)$ & $(20,17)$ \\
\hline & 20000 & $(3,2)$ & $(5,4)$ & $(7,6)$ & $(11,9)$ & $(14,12)$ & $(17,14)$ & $(19,17)$ \\
\hline & 30000 & $(3,2)$ & $(5,3)$ & $(7,5)$ & $(10,8)$ & $(13,11)$ & $(16,14)$ & $(19,16)$ \\
\hline & 40000 & $(3,2)$ & $(4,3)$ & $(6,5)$ & $(10,8)$ & $(13,11)$ & $(16,14)$ & $(18,16)$ \\
\hline & 50000 & $(3,2)$ & $(4,3)$ & $(6,5)$ & $(10,8)$ & $(13,11)$ & $(15,13)$ & $(18,16)$ \\
\hline \multirow[t]{5}{*}{750000} & 10000 & $(4,3)$ & $(6,4)$ & $(8,6)$ & $(12,9)$ & $(15,12)$ & $(18,15)$ & $(21,18)$ \\
\hline & 20000 & $(4,3)$ & $(5,4)$ & $(7,6)$ & $(11,9)$ & $(14,12)$ & $(17,15)$ & $(20,17)$ \\
\hline & 30000 & $(3,2)$ & $(5,4)$ & $(7,6)$ & $(11,9)$ & $(14,12)$ & $(17,14)$ & $(19,17)$ \\
\hline & 40000 & $(3,2)$ & $(5,4)$ & $(7,5)$ & $(10,9)$ & $(13,11)$ & $(16,14)$ & $(19,17)$ \\
\hline & 50000 & $(3,2)$ & $(4,3)$ & $(7,5)$ & $(10,8)$ & $(13,11)$ & $(16,14)$ & $(19,17)$ \\
\hline \multirow[t]{5}{*}{1000000} & 10000 & $(4,3)$ & $(6,4)$ & $(8,6)$ & $(12,10)$ & $(15,13)$ & $(18,15)$ & $(21,18)$ \\
\hline & 20000 & $(4,3)$ & $(5,4)$ & $(8,6)$ & $(11,9)$ & $(14,12)$ & $(17,15)$ & $(20,18)$ \\
\hline & 30000 & $(4,3)$ & $(5,4)$ & $(7,6)$ & $(11,9)$ & $(14,12)$ & $(17,15)$ & $(20,17)$ \\
\hline & 40000 & $(3,2)$ & $(5,4)$ & $(7,6)$ & $(11,9)$ & $(14,12)$ & $(17,15)$ & $(19,17)$ \\
\hline & 50000 & $(3,2)$ & $(5,4)$ & $(7,6)$ & $(10,9)$ & $(13,12)$ & $(16,14)$ & $(19,17)$ \\
\hline
\end{tabular}


assim como a taxa de atratividade do capital, considerada igual a $5 \%$ por período.

Como se pode perceber, a heurística implicou que todas as políticas adotadas devem seguir a política $(S, S-1)$, o que se mostra bastante razoável, pois a heurística é precisa no dimensionamento do nível de estoque máximo. Já com relação ao ponto de pedido, pode haver aumentos no custo de excesso e de ressuprimento sem que haja diminuição significativa no custo da falta. Entretanto, este aumento é relativamente baixo, o que torna aceitável a política definida pela heurística. Conforme mencionado anteriormente, essa aproximação fornece bons resultados, principalmente porque a diferença relativa entre os custos de falta e de excesso para as peças de reposição é muito alta e, dessa forma, o incremento nos níveis de estoque impacta, relativamente pouco, os custos totais.

Na Tabela 5 é apresentado um comparativo entre os custos totais e seus componentes principais para a heurística e a solução ótima. A diferença percentual média é de $2,65 \%$, sendo que a variação máxima verificada é de $13,78 \%$.

\section{Conclusões}

O estoque de peças de reposição está intimamente ligado ao balanço entre os custos da falta, do excesso e do ressuprimento, bem como à política de estoques adotada pela empresa. Nessa pesquisa, foi direcionada especial atenção para o caso de peças de reposição descartáveis, com política de estoques (S,s), cuja demanda fosse aderente à distribuição Poisson. Não foi considerada a variabilidade no lead-time de resposta, o que pode ser objeto de estudo em próximos trabalhos. Tampouco foram consideradas outras políticas de estoque.

Através do presente estudo, buscou-se modelar o consumo e a política de estoque de peças de reposição através de cadeias de Markov, um método estocástico que constitui uma alternativa computacionalmente mais eficiente que a simulação. Através de sua propriedade de convergência, é possível inferir a distribuição de probabilidades da posição de estoque, e a partir destes valores, determinar indicadores como estoque médio, probabilidade de falta e probabilidade de ressuprimento.

A heurística desenvolvida a partir desses resultados mostrou que a aproximação da política $(\mathrm{S}, \mathrm{s})$ pela $(\mathrm{S}, \mathrm{S}-1)$ é satisfatória em termos dos custos totais, sobretudo quando as peças de reposição apresentam elevado custo da falta, ou seja, extrema criticidade. Enquadram-se nessa categoria, por exemplo, empresas do setor de saúde, financeiro e de telecomunicações, nas quais a falta de estoques é passível de pesadas multas ou penalidades. 
Tabela 5. Comparação entre os custos.

\begin{tabular}{|c|c|c|c|c|c|c|c|c|c|c|}
\hline \multicolumn{5}{|c|}{ Parâmetros } & \multicolumn{6}{|c|}{ Solução ótima } \\
\hline $\begin{array}{l}\text { Media da } \\
\text { demanda }\end{array}$ & $\mathbf{M}$ & Caq & CR & $\mathbf{i}$ & $\mathbf{S}$ & $\mathbf{s}$ & Ressuprimento & Excesso & Falta & Total \\
\hline 0,5 & $\mathrm{R} \$ 250.000,00$ & $\mathrm{R} \$ 10.000,00$ & $\mathrm{R} \$ 800,00$ & $5 \%$ & 3 & 2 & $\mathrm{R} \$ 72,16$ & $\mathrm{R} \$ 1.250,97$ & $\mathrm{R} \$ 437,91$ & $\mathrm{R} \$ 1.761,04$ \\
\hline 0,5 & $\mathrm{R} \$ 250.000,00$ & $\mathrm{R} \$ 20.000,00$ & $\mathrm{R} \$ 800,00$ & $5 \%$ & 3 & 2 & $\mathrm{R} \$ 72,16$ & $\mathrm{R} \$ 2.501,94$ & $\mathrm{R} \$ 437,91$ & $\mathrm{R} \$ 3.012,01$ \\
\hline 0,5 & $\mathrm{R} \$ 250.000,00$ & $\mathrm{R} \$ 30.000,00$ & $\mathrm{R} \$ 800,00$ & $5 \%$ & 3 & 2 & $\mathrm{R} \$ 72,16$ & $\mathrm{R} \$ 3.752,91$ & $\mathrm{R} \$ 437,91$ & $\mathrm{R} \$ 4.262,98$ \\
\hline 0,5 & $\mathrm{R} \$ 250.000,00$ & $\mathrm{R} \$ 40.000,00$ & $\mathrm{R} \$ 800,00$ & $5 \%$ & 3 & 2 & $\mathrm{R} \$ 72,16$ & $\mathrm{R} \$ 5.003,88$ & $\mathrm{R} \$ 437,91$ & $\mathrm{R} \$ 5.513,95$ \\
\hline 0,5 & $\mathrm{R} \$ 250.000,00$ & $\mathrm{R} \$ 50.000,00$ & $\mathrm{R} \$ 800,00$ & $5 \%$ & 3 & 2 & $\mathrm{R} \$ 72,16$ & $\mathrm{R} \$ 6.254,85$ & $\mathrm{R} \$ 437,91$ & $\mathrm{R} \$ 6.764,92$ \\
\hline 0,5 & $\mathrm{R} \$ 500.000,00$ & $\mathrm{R} \$ 10.000,00$ & $\mathrm{R} \$ 800,00$ & $5 \%$ & 4 & 3 & $\mathrm{R} \$ 72,16$ & $\mathrm{R} \$ 1.750,09$ & $\mathrm{R} \$ 86,06$ & $\mathrm{R} \$ 1.908,31$ \\
\hline 0,5 & $\mathrm{R} \$ 500.000,00$ & $\mathrm{R} \$ 20.000,00$ & $\mathrm{R} \$ 800,00$ & $5 \%$ & 3 & 2 & $\mathrm{R} \$ 72,16$ & $\mathrm{R} \$ 2.501,94$ & $\mathrm{R} \$ 875,81$ & $\mathrm{R} \$ 3.449,91$ \\
\hline 0,5 & $\mathrm{R} \$ 500.000,00$ & $\mathrm{R} \$ 30.000,00$ & $\mathrm{R} \$ 800,00$ & $5 \%$ & 3 & 2 & $\mathrm{R} \$ 72,16$ & $\mathrm{R} \$ 3.752,91$ & $\mathrm{R} \$ 875,81$ & $\mathrm{R} \$ 4.700,88$ \\
\hline 0,5 & $\mathrm{R} \$ 500.000,00$ & $\mathrm{R} \$ 40.000,00$ & $\mathrm{R} \$ 800,00$ & $5 \%$ & 3 & 2 & $\mathrm{R} \$ 72,16$ & $\mathrm{R} \$ 5.003,88$ & $\mathrm{R} \$ 875,81$ & $\mathrm{R} \$ 5.951,85$ \\
\hline 0,5 & $\mathrm{R} \$ 500.000,00$ & $\mathrm{R} \$ 50.000,00$ & $\mathrm{R} \$ 800,00$ & $5 \%$ & 3 & 2 & $\mathrm{R} \$ 72,16$ & $\mathrm{R} \$ 6.254,85$ & $\mathrm{R} \$ 875,81$ & $\mathrm{R} \$ 7.202,82$ \\
\hline 0,5 & $\mathrm{R} \$ 750.000,00$ & $\mathrm{R} \$ 10.000,00$ & $\mathrm{R} \$ 800,00$ & $5 \%$ & 4 & 3 & $\mathrm{R} \$ 72,16$ & $\mathrm{R} \$ 1.750,09$ & $\mathrm{R} \$ 129,09$ & $\mathrm{R} \$ 1.951,34$ \\
\hline 0,5 & $\mathrm{R} \$ 750.000,00$ & $\mathrm{R} \$ 20.000,00$ & $\mathrm{R} \$ 800,00$ & $5 \%$ & 4 & 3 & $\mathrm{R} \$ 72,16$ & $\mathrm{R} \$ 3.500,19$ & $\mathrm{R} \$ 129,09$ & $\mathrm{R} \$ 3.701,44$ \\
\hline 0,5 & $\mathrm{R} \$ 750.000,00$ & $\mathrm{R} \$ 30.000,00$ & $\mathrm{R} \$ 800,00$ & $5 \%$ & 3 & 2 & $\mathrm{R} \$ 72,16$ & $\mathrm{R} \$ 3.752,91$ & $\mathrm{R} \$ 1.313,72$ & $\mathrm{R} \$ 5.138,79$ \\
\hline 0,5 & $\mathrm{R} \$ 750.000,00$ & $\mathrm{R} \$ 40.000,00$ & $\mathrm{R} \$ 800,00$ & $5 \%$ & 3 & 2 & $\mathrm{R} \$ 72,16$ & $\mathrm{R} \$ 5.003,88$ & $\mathrm{R} \$ 1.313,72$ & $\mathrm{R} \$ 6.389,76$ \\
\hline 0,5 & $\mathrm{R} \$ 750.000,00$ & $\mathrm{R} \$ 50.000,00$ & $\mathrm{R} \$ 800,00$ & $5 \%$ & 3 & 2 & $\mathrm{R} \$ 72,16$ & $\mathrm{R} \$ 6.254,85$ & $\mathrm{R} \$ 1.313,72$ & $\mathrm{R} \$ 7.640,73$ \\
\hline 0,5 & $\mathrm{R} \$ 1.000 .000,00$ & $\mathrm{R} \$ 10.000,00$ & $\mathrm{R} \$ 800,00$ & $5 \%$ & 4 & 3 & $\mathrm{R} \$ 72,16$ & $\mathrm{R} \$ 1.750,09$ & $\mathrm{R} \$ 172,12$ & $\mathrm{R} \$ 1.994,37$ \\
\hline 0,5 & $\mathrm{R} \$ 1.000 .000,00$ & $\mathrm{R} \$ 20.000,00$ & $\mathrm{R} \$ 800,00$ & $5 \%$ & 4 & 3 & $\mathrm{R} \$ 72,16$ & $\mathrm{R} \$ 3.500,19$ & $\mathrm{R} \$ 172,12$ & $\mathrm{R} \$ 3.744,47$ \\
\hline 0,5 & $\mathrm{R} \$ 1.000 .000,00$ & $\mathrm{R} \$ 30.000,00$ & $\mathrm{R} \$ 800,00$ & $5 \%$ & 4 & 3 & $\mathrm{R} \$ 72,16$ & $\mathrm{R} \$ 5.250,28$ & $\mathrm{R} \$ 172,12$ & $\mathrm{R} \$ 5.494,56$ \\
\hline 0,5 & $\mathrm{R} \$ 1.000 .000,00$ & $\mathrm{R} \$ 40.000,00$ & $\mathrm{R} \$ 800,00$ & $5 \%$ & 3 & 2 & $\mathrm{R} \$ 72,16$ & $\mathrm{R} \$ 5.003,88$ & $\mathrm{R} \$ 1.751,62$ & $\mathrm{R} \$ 6.827,66$ \\
\hline 0,5 & $\mathrm{R} \$ 1.000 .000,00$ & $\mathrm{R} \$ 50.000,00$ & $\mathrm{R} \$ 800,00$ & $5 \%$ & 3 & 2 & $\mathrm{R} \$ 72,16$ & $\mathrm{R} \$ 6.254,85$ & $\mathrm{R} \$ 1.751,62$ & $\mathrm{R} \$ 8.078,63$ \\
\hline 1 & $\mathrm{R} \$ 250.000,00$ & $\mathrm{R} \$ 10.000,00$ & $\mathrm{R} \$ 800,00$ & $5 \%$ & 5 & 4 & $\mathrm{R} \$ 211,39$ & $\mathrm{R} \$ 2.000,34$ & $\mathrm{R} \$ 148,55$ & $\mathrm{R} \$ 2.360,28$ \\
\hline 1 & $\mathrm{R} \$ 250.000,00$ & $\mathrm{R} \$ 20.000,00$ & $\mathrm{R} \$ 800,00$ & $5 \%$ & 4 & 3 & $\mathrm{R} \$ 211,39$ & $\mathrm{R} \$ 3.004,35$ & $\mathrm{R} \$ 914,96$ & $\mathrm{R} \$ 4.130,70$ \\
\hline 1 & $\mathrm{R} \$ 250.000,00$ & $\mathrm{R} \$ 30.000,00$ & $\mathrm{R} \$ 800,00$ & $5 \%$ & 4 & 3 & $\mathrm{R} \$ 211,39$ & $\mathrm{R} \$ 4.506,52$ & $\mathrm{R} \$ 914,96$ & $\mathrm{R} \$ 5.632,88$ \\
\hline 1 & $\mathrm{R} \$ 250.000,00$ & $\mathrm{R} \$ 40.000,00$ & $\mathrm{R} \$ 800,00$ & $5 \%$ & 4 & 3 & $\mathrm{R} \$ 211,39$ & $\mathrm{R} \$ 6.008,70$ & $\mathrm{R} \$ 914,96$ & $\mathrm{R} \$ 7.135,05$ \\
\hline 1 & $\mathrm{R} \$ 250.000,00$ & $\mathrm{R} \$ 50.000,00$ & $\mathrm{R} \$ 800,00$ & $5 \%$ & 4 & 3 & $\mathrm{R} \$ 211,39$ & $\mathrm{R} \$ 7.510,87$ & $\mathrm{R} \$ 914,96$ & $\mathrm{R} \$ 8.637,23$ \\
\hline 1 & $\mathrm{R} \$ 500.000,00$ & $\mathrm{R} \$ 10.000,00$ & $\mathrm{R} \$ 800,00$ & $5 \%$ & 5 & 4 & $\mathrm{R} \$ 211,39$ & $\mathrm{R} \$ 2.000,34$ & $\mathrm{R} \$ 297,09$ & $\mathrm{R} \$ 2.508,83$ \\
\hline 1 & $\mathrm{R} \$ 500.000,00$ & $\mathrm{R} \$ 20.000,00$ & $\mathrm{R} \$ 800,00$ & $5 \%$ & 5 & 4 & $\mathrm{R} \$ 211,39$ & $\mathrm{R} \$ 4.000,69$ & $\mathrm{R} \$ 297,09$ & $\mathrm{R} \$ 4.509,17$ \\
\hline 1 & $\mathrm{R} \$ 500.000,00$ & $\mathrm{R} \$ 30.000,00$ & $\mathrm{R} \$ 800,00$ & $5 \%$ & 5 & 3 & $\mathrm{R} \$ 118,38$ & $\mathrm{R} \$ 5.451,23$ & $\mathrm{R} \$ 860,99$ & $\mathrm{R} \$ 6.430,60$ \\
\hline 1 & $\mathrm{R} \$ 500.000,00$ & $\mathrm{R} \$ 40.000,00$ & $\mathrm{R} \$ 800,00$ & $5 \%$ & 4 & 3 & $\mathrm{R} \$ 211,39$ & $\mathrm{R} \$ 6.008,70$ & $\mathrm{R} \$ 1.829,92$ & $\mathrm{R} \$ 8.050,01$ \\
\hline 1 & $\mathrm{R} \$ 500.000,00$ & $\mathrm{R} \$ 50.000,00$ & $\mathrm{R} \$ 800,00$ & $5 \%$ & 4 & 3 & $\mathrm{R} \$ 211,39$ & $\mathrm{R} \$ 7.510,87$ & $\mathrm{R} \$ 1.829,92$ & $\mathrm{R} \$ 9.552,19$ \\
\hline 1 & $\mathrm{R} \$ 750.000,00$ & $\mathrm{R} \$ 10.000,00$ & $\mathrm{R} \$ 800,00$ & $5 \%$ & 6 & 4 & $\mathrm{R} \$ 118,38$ & $\mathrm{R} \$ 2.316,22$ & $\mathrm{R} \$ 203,41$ & $\mathrm{R} \$ 2.638,00$ \\
\hline 1 & $\mathrm{R} \$ 750.000,00$ & $\mathrm{R} \$ 20.000,00$ & $\mathrm{R} \$ 800,00$ & $5 \%$ & 5 & 4 & $\mathrm{R} \$ 211,39$ & $\mathrm{R} \$ 4.000,69$ & $\mathrm{R} \$ 445,64$ & $\mathrm{R} \$ 4.657,72$ \\
\hline 1 & $\mathrm{R} \$ 750.000,00$ & $\mathrm{R} \$ 30.000,00$ & $\mathrm{R} \$ 800,00$ & $5 \%$ & 5 & 4 & $\mathrm{R} \$ 211,39$ & $\mathrm{R} \$ 6.001,03$ & $\mathrm{R} \$ 445,64$ & $\mathrm{R} \$ 6.658,06$ \\
\hline 1 & $\mathrm{R} \$ 750.000,00$ & $\mathrm{R} \$ 40.000,00$ & $\mathrm{R} \$ 800,00$ & $5 \%$ & 5 & 4 & $\mathrm{R} \$ 211,39$ & $\mathrm{R} \$ 8.001,38$ & $\mathrm{R} \$ 445,64$ & $\mathrm{R} \$ 8.658,41$ \\
\hline 1 & $\mathrm{R} \$ 750.000,00$ & $\mathrm{R} \$ 50.000,00$ & $\mathrm{R} \$ 800,00$ & $5 \%$ & 4 & 3 & $\mathrm{R} \$ 211,39$ & $\mathrm{R} \$ 7.510,87$ & $\mathrm{R} \$ 2.744,89$ & $\mathrm{R} \$ 10.467,15$ \\
\hline 1 & $\mathrm{R} \$ 1.000 .000,00$ & $\mathrm{R} \$ 10.000,00$ & $\mathrm{R} \$ 800,00$ & $5 \%$ & 6 & 4 & $\mathrm{R} \$ 118,38$ & $\mathrm{R} \$ 2.316,22$ & $\mathrm{R} \$ 271,21$ & $\mathrm{R} \$ 2.705,80$ \\
\hline 1 & $\mathrm{R} \$ 1.000 .000,00$ & $\mathrm{R} \$ 20.000,00$ & $\mathrm{R} \$ 800,00$ & $5 \%$ & 5 & 4 & $\mathrm{R} \$ 211,39$ & $\mathrm{R} \$ 4.000,69$ & $\mathrm{R} \$ 594,18$ & $\mathrm{R} \$ 4.806,27$ \\
\hline 1 & $\mathrm{R} \$ 1.000 .000,00$ & $\mathrm{R} \$ 30.000,00$ & $\mathrm{R} \$ 800,00$ & $5 \%$ & 5 & 4 & $\mathrm{R} \$ 211,39$ & $\mathrm{R} \$ 6.001,03$ & $\mathrm{R} \$ 594,18$ & $\mathrm{R} \$ 6.806,61$ \\
\hline 1 & $\mathrm{R} \$ 1.000 .000,00$ & $\mathrm{R} \$ 40.000,00$ & $\mathrm{R} \$ 800,00$ & $5 \%$ & 5 & 4 & $\mathrm{R} \$ 211,39$ & $\mathrm{R} \$ 8.001,38$ & $\mathrm{R} \$ 594,18$ & $\mathrm{R} \$ 8.806,96$ \\
\hline 1 & $\mathrm{R} \$ 1.000 .000,00$ & $\mathrm{R} \$ 50.000,00$ & $\mathrm{R} \$ 800,00$ & $5 \%$ & 5 & 4 & $\mathrm{R} \$ 211,39$ & $\mathrm{R} \$ 10.001,72$ & $\mathrm{R} \$ 594,18$ & $\mathrm{R} \$ 10.807,30$ \\
\hline 2 & $\mathrm{R} \$ 250.000,00$ & $\mathrm{R} \$ 10.000,00$ & $\mathrm{R} \$ 800,00$ & $5 \%$ & 7 & 5 & $\mathrm{R} \$ 310,28$ & $\mathrm{R} \$ 2.382,03$ & $\mathrm{R} \$ 479,04$ & $\mathrm{R} \$ 3.171,35$ \\
\hline 2 & $\mathrm{R} \$ 250.000,00$ & $\mathrm{R} \$ 20.000,00$ & $\mathrm{R} \$ 800,00$ & $5 \%$ & 7 & 5 & $\mathrm{R} \$ 310,28$ & $\mathrm{R} \$ 4.764,07$ & $\mathrm{R} \$ 479,04$ & $\mathrm{R} \$ 5.553,38$ \\
\hline 2 & $\mathrm{R} \$ 250.000,00$ & $\mathrm{R} \$ 30.000,00$ & $\mathrm{R} \$ 800,00$ & $5 \%$ & 6 & 5 & $\mathrm{R} \$ 475,20$ & $\mathrm{R} \$ 6.008,89$ & $\mathrm{R} \$ 1.133,45$ & $\mathrm{R} \$ 7.617,53$ \\
\hline 2 & $\mathrm{R} \$ 250.000,00$ & $\mathrm{R} \$ 40.000,00$ & $\mathrm{R} \$ 800,00$ & $5 \%$ & 6 & 5 & $\mathrm{R} \$ 475,20$ & $\mathrm{R} \$ 8.011,85$ & $\mathrm{R} \$ 1.133,45$ & $\mathrm{R} \$ 9.620,50$ \\
\hline 2 & $\mathrm{R} \$ 250.000,00$ & $\mathrm{R} \$ 50.000,00$ & $\mathrm{R} \$ 800,00$ & $5 \%$ & 6 & 4 & $\mathrm{R} \$ 310,28$ & $\mathrm{R} \$ 9.428,67$ & $\mathrm{R} \$ 1.850,45$ & $\mathrm{R} \$ 11.589,40$ \\
\hline 2 & $\mathrm{R} \$ 500.000,00$ & $\mathrm{R} \$ 10.000,00$ & $\mathrm{R} \$ 800,00$ & $5 \%$ & 8 & 6 & $\mathrm{R} \$ 310,28$ & $\mathrm{R} \$ 2.881,07$ & $\mathrm{R} \$ 221,15$ & $\mathrm{R} \$ 3.412,51$ \\
\hline 2 & $\mathrm{R} \$ 500.000,00$ & $\mathrm{R} \$ 20.000,00$ & $\mathrm{R} \$ 800,00$ & $5 \%$ & 7 & 6 & $\mathrm{R} \$ 475,20$ & $\mathrm{R} \$ 5.001,39$ & $\mathrm{R} \$ 548,36$ & $\mathrm{R} \$ 6.024,95$ \\
\hline 2 & $\mathrm{R} \$ 500.000,00$ & $\mathrm{R} \$ 30.000,00$ & $\mathrm{R} \$ 800,00$ & $5 \%$ & 7 & 5 & $\mathrm{R} \$ 310,28$ & $\mathrm{R} \$ 7.146,10$ & $\mathrm{R} \$ 958,07$ & $\mathrm{R} \$ 8.414,45$ \\
\hline 2 & $\mathrm{R} \$ 500.000,00$ & $\mathrm{R} \$ 40.000,00$ & $\mathrm{R} \$ 800,00$ & $5 \%$ & 6 & 5 & $\mathrm{R} \$ 475,20$ & $\mathrm{R} \$ 8.011,85$ & $\mathrm{R} \$ 2.266,90$ & $\mathrm{R} \$ 10.753,95$ \\
\hline 2 & $\mathrm{R} \$ 500.000,00$ & $\mathrm{R} \$ 50.000,00$ & $\mathrm{R} \$ 800,00$ & $5 \%$ & 6 & 5 & $\mathrm{R} \$ 475,20$ & $\mathrm{R} \$ 10.014,81$ & $\mathrm{R} \$ 2.266,90$ & $\mathrm{R} \$ 12.756,91$ \\
\hline
\end{tabular}




\begin{tabular}{|c|c|c|c|c|c|c|}
\hline \multicolumn{6}{|c|}{ Heurística } & \multirow[t]{2}{*}{ Variação percentual } \\
\hline $\mathrm{S}^{\prime}$ & $\mathbf{s}^{\prime}$ & Ressuprimento & Excesso & Falta & Total & \\
\hline 3 & 3 & $\mathrm{R} \$ 314,78$ & $\mathrm{R} \$ 1.250,97$ & $\mathrm{R} \$ 437,91$ & $\mathrm{R} \$ 2.003,65$ & 13,78 \\
\hline 3 & 3 & $\mathrm{R} \$ 314,78$ & $\mathrm{R} \$ 2.501,94$ & $\mathrm{R} \$ 437,91$ & $\mathrm{R} \$ 3.254,62$ & 8,05 \\
\hline 3 & 3 & $\mathrm{R} \$ 314,78$ & $\mathrm{R} \$ 3.752,91$ & $\mathrm{R} \$ 437,91$ & $\mathrm{R} \$ 4.505,59$ & 5,69 \\
\hline 3 & 3 & $\mathrm{R} \$ 314,78$ & $\mathrm{R} \$ 5.003,88$ & $\mathrm{R} \$ 437,91$ & $\mathrm{R} \$ 5.756,56$ & 4,40 \\
\hline 3 & 3 & $\mathrm{R} \$ 314,78$ & $\mathrm{R} \$ 6.254,85$ & $\mathrm{R} \$ 437,91$ & $\mathrm{R} \$ 7.007,53$ & 3,59 \\
\hline 4 & 4 & $\mathrm{R} \$ 314,78$ & $\mathrm{R} \$ 1.750,09$ & $\mathrm{R} \$ 86,06$ & $\mathrm{R} \$ 2.150,93$ & 12,71 \\
\hline 3 & 3 & $\mathrm{R} \$ 314,78$ & $\mathrm{R} \$ 2.501,94$ & $\mathrm{R} \$ 875,81$ & $\mathrm{R} \$ 3.692,53$ & 7,03 \\
\hline 3 & 3 & $\mathrm{R} \$ 314,78$ & $\mathrm{R} \$ 3.752,91$ & $\mathrm{R} \$ 875,81$ & $\mathrm{R} \$ 4.943,50$ & 5,16 \\
\hline 3 & 3 & $\mathrm{R} \$ 314,78$ & $\mathrm{R} \$ 5.003,88$ & $\mathrm{R} \$ 875,81$ & $\mathrm{R} \$ 6.194,46$ & 4,08 \\
\hline 3 & 3 & $\mathrm{R} \$ 314,78$ & $\mathrm{R} \$ 6.254,85$ & $\mathrm{R} \$ 875,81$ & $\mathrm{R} \$ 7.445,43$ & 3,37 \\
\hline 4 & 4 & $\mathrm{R} \$ 314,78$ & $\mathrm{R} \$ 1.750,09$ & $\mathrm{R} \$ 129,09$ & $\mathrm{R} \$ 2.193,96$ & 12,43 \\
\hline 4 & 4 & $\mathrm{R} \$ 314,78$ & $\mathrm{R} \$ 3.500,19$ & $\mathrm{R} \$ 129,09$ & $\mathrm{R} \$ 3.944,05$ & 6,55 \\
\hline 3 & 3 & $\mathrm{R} \$ 314,78$ & $\mathrm{R} \$ 3.752,91$ & $\mathrm{R} \$ 1.313,72$ & $\mathrm{R} \$ 5.381,40$ & 4,72 \\
\hline 3 & 3 & $\mathrm{R} \$ 314,78$ & $\mathrm{R} \$ 5.003,88$ & $\mathrm{R} \$ 1.313,72$ & $\mathrm{R} \$ 6.632,37$ & 3,80 \\
\hline 3 & 3 & $\mathrm{R} \$ 314,78$ & $\mathrm{R} \$ 6.254,85$ & $\mathrm{R} \$ 1.313,72$ & $\mathrm{R} \$ 7.883,34$ & 3,18 \\
\hline 4 & 4 & $\mathrm{R} \$ 314,78$ & $\mathrm{R} \$ 1.750,09$ & $\mathrm{R} \$ 172,12$ & $\mathrm{R} \$ 2.236,98$ & 12,16 \\
\hline 4 & 4 & $\mathrm{R} \$ 314,78$ & $\mathrm{R} \$ 3.500,19$ & $\mathrm{R} \$ 172,12$ & $\mathrm{R} \$ 3.987,08$ & 6,48 \\
\hline 4 & 4 & $\mathrm{R} \$ 314,78$ & $\mathrm{R} \$ 5.250,28$ & $\mathrm{R} \$ 172,12$ & $\mathrm{R} \$ 5.737,17$ & 4,42 \\
\hline 3 & 3 & $\mathrm{R} \$ 314,78$ & $\mathrm{R} \$ 5.003,88$ & $\mathrm{R} \$ 1.751,62$ & $\mathrm{R} \$ 7.070,28$ & 3,55 \\
\hline 3 & 3 & $\mathrm{R} \$ 314,78$ & $\mathrm{R} \$ 6.254,85$ & $\mathrm{R} \$ 1.751,62$ & $\mathrm{R} \$ 8.321,25$ & 3,00 \\
\hline 5 & 5 & $\mathrm{R} \$ 505,70$ & $\mathrm{R} \$ 2.000,34$ & $\mathrm{R} \$ 148,55$ & $R \$ 2.654,59$ & 12,47 \\
\hline 4 & 4 & $\mathrm{R} \$ 505,70$ & $\mathrm{R} \$ 3.004,35$ & $\mathrm{R} \$ 914,96$ & $\mathrm{R} \$ 4.425,01$ & 7,12 \\
\hline 4 & 4 & $\mathrm{R} \$ 505,70$ & $\mathrm{R} \$ 4.506,52$ & $\mathrm{R} \$ 914,96$ & $\mathrm{R} \$ 5.927,18$ & 5,22 \\
\hline 4 & 4 & $\mathrm{R} \$ 505,70$ & $\mathrm{R} \$ 6.008,70$ & $\mathrm{R} \$ 914,96$ & $\mathrm{R} \$ 7.429,36$ & 4,12 \\
\hline 4 & 4 & $\mathrm{R} \$ 505,70$ & $\mathrm{R} \$ 7.510,87$ & $\mathrm{R} \$ 914,96$ & $\mathrm{R} \$ 8.931,53$ & 3,41 \\
\hline 5 & 5 & $\mathrm{R} \$ 505,70$ & $\mathrm{R} \$ 2.000,34$ & $\mathrm{R} \$ 297,09$ & $\mathrm{R} \$ 2.803,13$ & 11,73 \\
\hline 5 & 5 & $\mathrm{R} \$ 505,70$ & $\mathrm{R} \$ 4.000,69$ & $\mathrm{R} \$ 297,09$ & $\mathrm{R} \$ 4.803,48$ & 6,53 \\
\hline 5 & 5 & $\mathrm{R} \$ 505,70$ & $\mathrm{R} \$ 6.001,03$ & $\mathrm{R} \$ 297,09$ & $\mathrm{R} \$ 6.803,82$ & 5,80 \\
\hline 4 & 4 & $\mathrm{R} \$ 505,70$ & $\mathrm{R} \$ 6.008,70$ & $\mathrm{R} \$ 1.829,92$ & $\mathrm{R} \$ 8.344,32$ & 3,66 \\
\hline 4 & 4 & $\mathrm{R} \$ 505,70$ & $\mathrm{R} \$ 7.510,87$ & $\mathrm{R} \$ 1.829,92$ & $\mathrm{R} \$ 9.846,49$ & 3,08 \\
\hline 5 & 5 & $\mathrm{R} \$ 505,70$ & $\mathrm{R} \$ 2.000,34$ & $\mathrm{R} \$ 445,64$ & $\mathrm{R} \$ 2.951,68$ & 11,89 \\
\hline 5 & 5 & $\mathrm{R} \$ 505,70$ & $\mathrm{R} \$ 4.000,69$ & $\mathrm{R} \$ 445,64$ & $\mathrm{R} \$ 4.952,02$ & 6,32 \\
\hline 5 & 5 & $\mathrm{R} \$ 505,70$ & $\mathrm{R} \$ 6.001,03$ & $\mathrm{R} \$ 445,64$ & $\mathrm{R} \$ 6.952,37$ & 4,42 \\
\hline 5 & 5 & $\mathrm{R} \$ 505,70$ & $\mathrm{R} \$ 8.001,38$ & $\mathrm{R} \$ 445,64$ & $\mathrm{R} \$ 8.952,71$ & 3,40 \\
\hline 4 & 4 & $\mathrm{R} \$ 505,70$ & $\mathrm{R} \$ 7.510,87$ & $\mathrm{R} \$ 2.744,89$ & $\mathrm{R} \$ 10.761,45$ & 2,81 \\
\hline 6 & 6 & $\mathrm{R} \$ 505,70$ & $\mathrm{R} \$ 2.500,05$ & $\mathrm{R} \$ 83,24$ & $\mathrm{R} \$ 3.088,98$ & 14,16 \\
\hline 5 & 5 & $\mathrm{R} \$ 505,70$ & $\mathrm{R} \$ 4.000,69$ & $\mathrm{R} \$ 594,18$ & $\mathrm{R} \$ 5.100,57$ & 6,12 \\
\hline 5 & 5 & $\mathrm{R} \$ 505,70$ & $\mathrm{R} \$ 6.001,03$ & $\mathrm{R} \$ 594,18$ & $\mathrm{R} \$ 7.100,91$ & 4,32 \\
\hline 5 & 5 & $\mathrm{R} \$ 505,70$ & $\mathrm{R} \$ 8.001,38$ & $\mathrm{R} \$ 594,18$ & $\mathrm{R} \$ 9.101,26$ & 3,34 \\
\hline 5 & 5 & $\mathrm{R} \$ 505,70$ & $\mathrm{R} \$ 10.001,72$ & $\mathrm{R} \$ 594,18$ & $\mathrm{R} \$ 11.101,60$ & 2,72 \\
\hline 7 & 7 & $\mathrm{R} \$ 691,73$ & $\mathrm{R} \$ 2.500,70$ & $\mathrm{R} \$ 274,18$ & $\mathrm{R} \$ 3.466,61$ & 9,31 \\
\hline 6 & 6 & $\mathrm{R} \$ 691,73$ & $\mathrm{R} \$ 4.005,92$ & $\mathrm{R} \$ 1.133,45$ & $\mathrm{R} \$ 5.831,11$ & 5,00 \\
\hline 6 & 6 & $\mathrm{R} \$ 691,73$ & $\mathrm{R} \$ 6.008,89$ & $\mathrm{R} \$ 1.133,45$ & $\mathrm{R} \$ 7.834,07$ & 2,84 \\
\hline 6 & 6 & $\mathrm{R} \$ 691,73$ & $\mathrm{R} \$ 8.011,85$ & $\mathrm{R} \$ 1.133,45$ & $\mathrm{R} \$ 9.837,03$ & 2,25 \\
\hline 6 & 6 & $\mathrm{R} \$ 691,73$ & $\mathrm{R} \$ 10.014,81$ & $\mathrm{R} \$ 1.133,45$ & $\mathrm{R} \$ 11.839,99$ & 2,16 \\
\hline 7 & 7 & $\mathrm{R} \$ 691,73$ & $\mathrm{R} \$ 2.500,70$ & $\mathrm{R} \$ 548,36$ & $\mathrm{R} \$ 3.740,79$ & 9,62 \\
\hline 7 & 7 & $\mathrm{R} \$ 691,73$ & $\mathrm{R} \$ 5.001,39$ & $\mathrm{R} \$ 548,36$ & $\mathrm{R} \$ 6.241,48$ & 3,59 \\
\hline 7 & 7 & $\mathrm{R} \$ 691,73$ & $\mathrm{R} \$ 7.502,09$ & $\mathrm{R} \$ 548,36$ & $\mathrm{R} \$ 8.742,18$ & 3,89 \\
\hline 6 & 6 & $\mathrm{R} \$ 691,73$ & $\mathrm{R} \$ 8.011,85$ & $\mathrm{R} \$ 2.266,90$ & $\mathrm{R} \$ 10.970,48$ & 2,01 \\
\hline 6 & 6 & $\mathrm{R} \$ 691,73$ & $\mathrm{R} \$ 10.014,81$ & $\mathrm{R} \$ 2.266,90$ & $\mathrm{R} \$ 12.973,45$ & 1,70 \\
\hline
\end{tabular}


Tabela 7. Continuação...

\begin{tabular}{|c|c|c|c|c|c|c|c|c|c|c|}
\hline \multicolumn{5}{|c|}{ Parâmetros } & \multicolumn{6}{|c|}{ Solução ótima } \\
\hline $\begin{array}{l}\text { Media da } \\
\text { demanda }\end{array}$ & $\mathbf{M}$ & Caq & CR & i & $\mathbf{S}$ & $\mathbf{s}$ & Ressuprimento & Excesso & Falta & Total \\
\hline 2 & $\mathrm{R} \$ 750.000,00$ & $\mathrm{R} \$ 10.000,00$ & $\mathrm{R} \$ 800,00$ & $5 \%$ & 8 & 6 & $\mathrm{R} \$ 310,28$ & $\mathrm{R} \$ 2.881,07$ & $\mathrm{R} \$ 331,73$ & $\mathrm{R} \$ 3.523,08$ \\
\hline 2 & $\mathrm{R} \$ 750.000,00$ & $\mathrm{R} \$ 20.000,00$ & $\mathrm{R} \$ 800,00$ & $5 \%$ & 7 & 6 & $\mathrm{R} \$ 475,20$ & $\mathrm{R} \$ 5.001,39$ & $\mathrm{R} \$ 822,54$ & $\mathrm{R} \$ 6.299,13$ \\
\hline 2 & $\mathrm{R} \$ 750.000,00$ & $\mathrm{R} \$ 30.000,00$ & $\mathrm{R} \$ 800,00$ & $5 \%$ & 7 & 6 & $\mathrm{R} \$ 475,20$ & $\mathrm{R} \$ 7.502,09$ & $\mathrm{R} \$ 822,54$ & $\mathrm{R} \$ 8.799,82$ \\
\hline 2 & $\mathrm{R} \$ 750.000,00$ & $\mathrm{R} \$ 40.000,00$ & $\mathrm{R} \$ 800,00$ & $5 \%$ & 7 & 5 & $\mathrm{R} \$ 310,28$ & $\mathrm{R} \$ 9.528,13$ & $\mathrm{R} \$ 1.437,11$ & $\mathrm{R} \$ 11.275,52$ \\
\hline 2 & $\mathrm{R} \$ 750.000,00$ & $\mathrm{R} \$ 50.000,00$ & $\mathrm{R} \$ 800,00$ & $5 \%$ & 7 & 5 & $\mathrm{R} \$ 310,28$ & $\mathrm{R} \$ 11.910,16$ & $\mathrm{R} \$ 1.437,11$ & $\mathrm{R} \$ 13.657,55$ \\
\hline 2 & $\mathrm{R} \$ 1.000 .000,00$ & $\mathrm{R} \$ 10.000,00$ & $\mathrm{R} \$ 800,00$ & $5 \%$ & 8 & 6 & $\mathrm{R} \$ 310,28$ & $\mathrm{R} \$ 2.881,07$ & $\mathrm{R} \$ 442,30$ & $\mathrm{R} \$ 3.633,66$ \\
\hline 2 & $\mathrm{R} \$ 1.000 .000,00$ & $\mathrm{R} \$ 20.000,00$ & $\mathrm{R} \$ 800,00$ & $5 \%$ & 8 & 6 & $\mathrm{R} \$ 310,28$ & $\mathrm{R} \$ 5.762,15$ & $\mathrm{R} \$ 442,30$ & $\mathrm{R} \$ 6.514,73$ \\
\hline 2 & $\mathrm{R} \$ 1.000 .000,00$ & $\mathrm{R} \$ 30.000,00$ & $\mathrm{R} \$ 800,00$ & $5 \%$ & 7 & 6 & $\mathrm{R} \$ 475,20$ & $\mathrm{R} \$ 7.502,09$ & $\mathrm{R} \$ 1.096,72$ & $\mathrm{R} \$ 9.074,00$ \\
\hline 2 & $\mathrm{R} \$ 1.000 .000,00$ & $\mathrm{R} \$ 40.000,00$ & $\mathrm{R} \$ 800,00$ & $5 \%$ & 7 & 6 & $\mathrm{R} \$ 475,20$ & $\mathrm{R} \$ 10.002,78$ & $\mathrm{R} \$ 1.096,72$ & $\mathrm{R} \$ 11.574,70$ \\
\hline 2 & $\mathrm{R} \$ 1.000 .000,00$ & $\mathrm{R} \$ 50.000,00$ & $\mathrm{R} \$ 800,00$ & $5 \%$ & 7 & 6 & $\mathrm{R} \$ 475,20$ & $\mathrm{R} \$ 12.503,48$ & $\mathrm{R} \$ 1.096,72$ & $\mathrm{R} \$ 14.075,39$ \\
\hline 4 & $\mathrm{R} \$ 250.000,00$ & $\mathrm{R} \$ 10.000,00$ & $\mathrm{R} \$ 800,00$ & $5 \%$ & 11 & 8 & $\mathrm{R} \$ 497,16$ & $\mathrm{R} \$ 3.345,14$ & $\mathrm{R} \$ 485,23$ & $\mathrm{R} \$ 4.327,52$ \\
\hline 4 & $\mathrm{R} \$ 250.000,00$ & $\mathrm{R} \$ 20.000,00$ & $\mathrm{R} \$ 800,00$ & $5 \%$ & 10 & 8 & $\mathrm{R} \$ 617,66$ & $\mathrm{R} \$ 5.935,25$ & $\mathrm{R} \$ 801,83$ & $\mathrm{R} \$ 7.354,74$ \\
\hline 4 & $\mathrm{R} \$ 250.000,00$ & $\mathrm{R} \$ 30.000,00$ & $\mathrm{R} \$ 800,00$ & $5 \%$ & 9 & 8 & $\mathrm{R} \$ 726,74$ & $\mathrm{R} \$ 7.518,40$ & $\mathrm{R} \$ 2.033,06$ & $\mathrm{R} \$ 10.278,19$ \\
\hline 4 & $\mathrm{R} \$ 250.000,00$ & $\mathrm{R} \$ 40.000,00$ & $\mathrm{R} \$ 800,00$ & $5 \%$ & 9 & 7 & $\mathrm{R} \$ 617,66$ & $\mathrm{R} \$ 9.888,60$ & $\mathrm{R} \$ 2.262,78$ & $\mathrm{R} \$ 12.769,04$ \\
\hline 4 & $\mathrm{R} \$ 250.000,00$ & $\mathrm{R} \$ 50.000,00$ & $\mathrm{R} \$ 800,00$ & $5 \%$ & 9 & 7 & $\mathrm{R} \$ 617,66$ & $\mathrm{R} \$ 12.360,75$ & $\mathrm{R} \$ 2.262,78$ & $\mathrm{R} \$ 15.241,19$ \\
\hline 4 & $\mathrm{R} \$ 500.000,00$ & $\mathrm{R} \$ 10.000,00$ & $\mathrm{R} \$ 800,00$ & $5 \%$ & 11 & 9 & $\mathrm{R} \$ 617,66$ & $\mathrm{R} \$ 3.466,02$ & $\mathrm{R} \$ 524,44$ & $\mathrm{R} \$ 4.608,12$ \\
\hline 4 & $\mathrm{R} \$ 500.000,00$ & $\mathrm{R} \$ 20.000,00$ & $\mathrm{R} \$ 800,00$ & $5 \%$ & 11 & 9 & $\mathrm{R} \$ 617,66$ & $\mathrm{R} \$ 6.932,04$ & $\mathrm{R} \$ 524,44$ & $\mathrm{R} \$ 8.074,14$ \\
\hline 4 & $\mathrm{R} \$ 500.000,00$ & $\mathrm{R} \$ 30.000,00$ & $\mathrm{R} \$ 800,00$ & $5 \%$ & 10 & 8 & $\mathrm{R} \$ 617,66$ & $\mathrm{R} \$ 8.902,87$ & $\mathrm{R} \$ 1.603,66$ & $\mathrm{R} \$ 11.124,19$ \\
\hline 4 & $\mathrm{R} \$ 500.000,00$ & $\mathrm{R} \$ 40.000,00$ & $\mathrm{R} \$ 800,00$ & $5 \%$ & 10 & 8 & $\mathrm{R} \$ 617,66$ & $\mathrm{R} \$ 11.870,50$ & $\mathrm{R} \$ 1.603,66$ & $\mathrm{R} \$ 14.091,81$ \\
\hline 4 & $\mathrm{R} \$ 500.000,00$ & $\mathrm{R} \$ 50.000,00$ & $\mathrm{R} \$ 800,00$ & $5 \%$ & 10 & 8 & $\mathrm{R} \$ 617,66$ & $\mathrm{R} \$ 14.838,12$ & $\mathrm{R} \$ 1.603,66$ & $\mathrm{R} \$ 17.059,44$ \\
\hline 4 & $\mathrm{R} \$ 750.000,00$ & $\mathrm{R} \$ 10.000,00$ & $\mathrm{R} \$ 800,00$ & $5 \%$ & 12 & 9 & $\mathrm{R} \$ 497,16$ & $\mathrm{R} \$ 3.844,17$ & $\mathrm{R} \$ 476,85$ & $\mathrm{R} \$ 4.818,18$ \\
\hline 4 & $\mathrm{R} \$ 750.000,00$ & $\mathrm{R} \$ 20.000,00$ & $\mathrm{R} \$ 800,00$ & $5 \%$ & 11 & 9 & $\mathrm{R} \$ 617,66$ & $\mathrm{R} \$ 6.932,04$ & $\mathrm{R} \$ 786,66$ & $\mathrm{R} \$ 8.336,36$ \\
\hline 4 & $\mathrm{R} \$ 750.000,00$ & $\mathrm{R} \$ 30.000,00$ & $\mathrm{R} \$ 800,00$ & $5 \%$ & 11 & 9 & $\mathrm{R} \$ 617,66$ & $\mathrm{R} \$ 10.398,06$ & $\mathrm{R} \$ 786,66$ & $\mathrm{R} \$ 11.802,38$ \\
\hline 4 & $\mathrm{R} \$ 750.000,00$ & $\mathrm{R} \$ 40.000,00$ & $\mathrm{R} \$ 800,00$ & $5 \%$ & 10 & 9 & $\mathrm{R} \$ 726,74$ & $\mathrm{R} \$ 12.008,26$ & $\mathrm{R} \$ 2.129,82$ & $\mathrm{R} \$ 14.864,82$ \\
\hline 4 & $\mathrm{R} \$ 750.000,00$ & $\mathrm{R} \$ 50.000,00$ & $\mathrm{R} \$ 800,00$ & $5 \%$ & 10 & 8 & $\mathrm{R} \$ 617,66$ & $\mathrm{R} \$ 14.838,12$ & $\mathrm{R} \$ 2.405,48$ & $\mathrm{R} \$ 17.861,26$ \\
\hline 4 & $\mathrm{R} \$ 1.000 .000,00$ & $\mathrm{R} \$ 10.000,00$ & $\mathrm{R} \$ 800,00$ & $5 \%$ & 12 & 10 & $\mathrm{R} \$ 617,66$ & $\mathrm{R} \$ 3.965,50$ & $\mathrm{R} \$ 318,27$ & $\mathrm{R} \$ 4.901,42$ \\
\hline 4 & $\mathrm{R} \$ 1.000 .000,00$ & $\mathrm{R} \$ 20.000,00$ & $\mathrm{R} \$ 800,00$ & $5 \%$ & 11 & 9 & $\mathrm{R} \$ 617,66$ & $\mathrm{R} \$ 6.932,04$ & $\mathrm{R} \$ 1.048,88$ & $\mathrm{R} \$ 8.598,58$ \\
\hline 4 & $\mathrm{R} \$ 1.000 .000,00$ & $\mathrm{R} \$ 30.000,00$ & $\mathrm{R} \$ 800,00$ & $5 \%$ & 11 & 9 & $\mathrm{R} \$ 617,66$ & $\mathrm{R} \$ 10.398,06$ & $\mathrm{R} \$ 1.048,88$ & $\mathrm{R} \$ 12.064,60$ \\
\hline 4 & $\mathrm{R} \$ 1.000 .000,00$ & $\mathrm{R} \$ 40.000,00$ & $\mathrm{R} \$ 800,00$ & $5 \%$ & 11 & 9 & $\mathrm{R} \$ 617,66$ & $\mathrm{R} \$ 13.864,08$ & $\mathrm{R} \$ 1.048,88$ & $\mathrm{R} \$ 15.530,62$ \\
\hline 4 & $\mathrm{R} \$ 1.000 .000,00$ & $\mathrm{R} \$ 50.000,00$ & $\mathrm{R} \$ 800,00$ & $5 \%$ & 10 & 9 & $\mathrm{R} \$ 726,74$ & $\mathrm{R} \$ 15.010,33$ & $\mathrm{R} \$ 2.839,77$ & $\mathrm{R} \$ 18.576,83$ \\
\hline 6 & $\mathrm{R} \$ 250.000,00$ & $\mathrm{R} \$ 10.000,00$ & $\mathrm{R} \$ 800,00$ & $5 \%$ & 14 & 11 & $\mathrm{R} \$ 684,58$ & $\mathrm{R} \$ 3.951,93$ & $\mathrm{R} \$ 436,67$ & $\mathrm{R} \$ 5.073,18$ \\
\hline 6 & $\mathrm{R} \$ 250.000,00$ & $\mathrm{R} \$ 20.000,00$ & $\mathrm{R} \$ 800,00$ & $5 \%$ & 13 & 11 & $\mathrm{R} \$ 750,95$ & $\mathrm{R} \$ 6.991,23$ & $\mathrm{R} \$ 926,22$ & $\mathrm{R} \$ 8.668,40$ \\
\hline 6 & $\mathrm{R} \$ 250.000,00$ & $\mathrm{R} \$ 30.000,00$ & $\mathrm{R} \$ 800,00$ & $5 \%$ & 12 & 10 & $\mathrm{R} \$ 750,95$ & $\mathrm{R} \$ 9.000,34$ & $\mathrm{R} \$ 2.248,24$ & $\mathrm{R} \$ 11.999,53$ \\
\hline 6 & $\mathrm{R} \$ 250.000,00$ & $\mathrm{R} \$ 40.000,00$ & $\mathrm{R} \$ 800,00$ & $5 \%$ & 12 & 10 & $\mathrm{R} \$ 750,95$ & $\mathrm{R} \$ 12.000,45$ & $\mathrm{R} \$ 2.248,24$ & $\mathrm{R} \$ 14.999,64$ \\
\hline 6 & $\mathrm{R} \$ 250.000,00$ & $\mathrm{R} \$ 50.000,00$ & $\mathrm{R} \$ 800,00$ & $5 \%$ & 12 & 10 & $\mathrm{R} \$ 750,95$ & $\mathrm{R} \$ 15.000,57$ & $\mathrm{R} \$ 2.248,24$ & $\mathrm{R} \$ 17.999,76$ \\
\hline 6 & $\mathrm{R} \$ 500.000,00$ & $\mathrm{R} \$ 10.000,00$ & $\mathrm{R} \$ 800,00$ & $5 \%$ & 14 & 12 & $\mathrm{R} \$ 750,95$ & $\mathrm{R} \$ 3.993,76$ & $\mathrm{R} \$ 716,54$ & $\mathrm{R} \$ 5.461,26$ \\
\hline 6 & $\mathrm{R} \$ 500.000,00$ & $\mathrm{R} \$ 20.000,00$ & $\mathrm{R} \$ 800,00$ & $5 \%$ & 14 & 12 & $\mathrm{R} \$ 750,95$ & $\mathrm{R} \$ 7.987,53$ & $\mathrm{R} \$ 716,54$ & $\mathrm{R} \$ 9.455,02$ \\
\hline 6 & $\mathrm{R} \$ 500.000,00$ & $\mathrm{R} \$ 30.000,00$ & $\mathrm{R} \$ 800,00$ & $5 \%$ & 13 & 11 & $\mathrm{R} \$ 750,95$ & $\mathrm{R} \$ 10.486,85$ & $\mathrm{R} \$ 1.852,43$ & $\mathrm{R} \$ 13.090,23$ \\
\hline 6 & $\mathrm{R} \$ 500.000,00$ & $\mathrm{R} \$ 40.000,00$ & $\mathrm{R} \$ 800,00$ & $5 \%$ & 13 & 11 & $\mathrm{R} \$ 750,95$ & $\mathrm{R} \$ 13.982,47$ & $\mathrm{R} \$ 1.852,43$ & $\mathrm{R} \$ 16.585,85$ \\
\hline 6 & $\mathrm{R} \$ 500.000,00$ & $\mathrm{R} \$ 50.000,00$ & $\mathrm{R} \$ 800,00$ & $5 \%$ & 13 & 11 & $\mathrm{R} \$ 750,95$ & $\mathrm{R} \$ 17.478,08$ & $\mathrm{R} \$ 1.852,43$ & $\mathrm{R} \$ 20.081,47$ \\
\hline 6 & $\mathrm{R} \$ 750.000,00$ & $\mathrm{R} \$ 10.000,00$ & $\mathrm{R} \$ 800,00$ & $5 \%$ & 15 & 12 & $\mathrm{R} \$ 684,58$ & $\mathrm{R} \$ 4.451,05$ & $\mathrm{R} \$ 490,45$ & $\mathrm{R} \$ 5.626,09$ \\
\hline 6 & $\mathrm{R} \$ 750.000,00$ & $\mathrm{R} \$ 20.000,00$ & $\mathrm{R} \$ 800,00$ & $5 \%$ & 14 & 12 & $\mathrm{R} \$ 750,95$ & $\mathrm{R} \$ 7.987,53$ & $\mathrm{R} \$ 1.074,81$ & $\mathrm{R} \$ 9.813,29$ \\
\hline 6 & $\mathrm{R} \$ 750.000,00$ & $\mathrm{R} \$ 30.000,00$ & $\mathrm{R} \$ 800,00$ & $5 \%$ & 14 & 12 & $\mathrm{R} \$ 750,95$ & $\mathrm{R} \$ 11.981,29$ & $\mathrm{R} \$ 1.074,81$ & $\mathrm{R} \$ 13.807,06$ \\
\hline 6 & $\mathrm{R} \$ 750.000,00$ & $\mathrm{R} \$ 40.000,00$ & $\mathrm{R} \$ 800,00$ & $5 \%$ & 13 & 11 & $\mathrm{R} \$ 750,95$ & $\mathrm{R} \$ 13.982,47$ & $\mathrm{R} \$ 2.778,65$ & $\mathrm{R} \$ 17.512,07$ \\
\hline 6 & $\mathrm{R} \$ 750.000,00$ & $\mathrm{R} \$ 50.000,00$ & $\mathrm{R} \$ 800,00$ & $5 \%$ & 13 & 11 & $\mathrm{R} \$ 750,95$ & $\mathrm{R} \$ 17.478,08$ & $\mathrm{R} \$ 2.778,65$ & $\mathrm{R} \$ 21.007,68$ \\
\hline 6 & $\mathrm{R} \$ 1.000 .000,00$ & $\mathrm{R} \$ 10.000,00$ & $\mathrm{R} \$ 800,00$ & $5 \%$ & 15 & 13 & $\mathrm{R} \$ 750,95$ & $\mathrm{R} \$ 4.493,05$ & $\mathrm{R} \$ 522,19$ & $\mathrm{R} \$ 5.766,19$ \\
\hline 6 & $\mathrm{R} \$ 1.000 .000,00$ & $\mathrm{R} \$ 20.000,00$ & $\mathrm{R} \$ 800,00$ & $5 \%$ & 14 & 12 & $\mathrm{R} \$ 750,95$ & $\mathrm{R} \$ 7.987,53$ & $\mathrm{R} \$ 1.433,09$ & $\mathrm{R} \$ 10.171,56$ \\
\hline 6 & $\mathrm{R} \$ 1.000 .000,00$ & $\mathrm{R} \$ 30.000,00$ & $\mathrm{R} \$ 800,00$ & $5 \%$ & 14 & 12 & $\mathrm{R} \$ 750,95$ & $\mathrm{R} \$ 11.981,29$ & $\mathrm{R} \$ 1.433,09$ & $\mathrm{R} \$ 14.165,33$ \\
\hline 6 & $\mathrm{R} \$ 1.000 .000,00$ & $\mathrm{R} \$ 40.000,00$ & $\mathrm{R} \$ 800,00$ & $5 \%$ & 14 & 12 & $\mathrm{R} \$ 750,95$ & $\mathrm{R} \$ 15.975,06$ & $\mathrm{R} \$ 1.433,09$ & $\mathrm{R} \$ 18.159,09$ \\
\hline 6 & $\mathrm{R} \$ 1.000 .000,00$ & $\mathrm{R} \$ 50.000,00$ & $\mathrm{R} \$ 800,00$ & $5 \%$ & 13 & 12 & $\mathrm{R} \$ 786,12$ & $\mathrm{R} \$ 17.514,49$ & $\mathrm{R} \$ 3.628,49$ & $\mathrm{R} \$ 21.929,10$ \\
\hline
\end{tabular}


Heurística

Variação percentual

\begin{tabular}{|c|c|c|c|c|c|c|}
\hline $\mathbf{S}^{\prime}$ & $\mathrm{s}^{\prime}$ & Ressuprimento & Excesso & Falta & Total & \\
\hline 8 & 8 & $\mathrm{R} \$ 691,73$ & $\mathrm{R} \$ 3.000,15$ & $\mathrm{R} \$ 178,09$ & $\mathrm{R} \$ 3.869,96$ & 9,85 \\
\hline 7 & 7 & $\mathrm{R} \$ 691,73$ & $\mathrm{R} \$ 5.001,39$ & $\mathrm{R} \$ 822,54$ & $\mathrm{R} \$ 6.515,66$ & 3,44 \\
\hline 7 & 7 & $\mathrm{R} \$ 691,73$ & $\mathrm{R} \$ 7.502,09$ & $\mathrm{R} \$ 822,54$ & $\mathrm{R} \$ 9.016,36$ & 2,46 \\
\hline 7 & 7 & $\mathrm{R} \$ 691,73$ & $\mathrm{R} \$ 10.002,78$ & $\mathrm{R} \$ 822,54$ & $\mathrm{R} \$ 11.517,05$ & 2,14 \\
\hline 7 & 7 & $\mathrm{R} \$ 691,73$ & $\mathrm{R} \$ 12.503,48$ & $\mathrm{R} \$ 822,54$ & $\mathrm{R} \$ 14.017,75$ & 2,64 \\
\hline 8 & 8 & $\mathrm{R} \$ 691,73$ & $\mathrm{R} \$ 3.000,15$ & $\mathrm{R} \$ 237,45$ & $\mathrm{R} \$ 3.929,33$ & 8,14 \\
\hline 7 & 7 & $\mathrm{R} \$ 691,73$ & $\mathrm{R} \$ 5.001,39$ & $\mathrm{R} \$ 1.096,72$ & $\mathrm{R} \$ 6.789,84$ & 4,22 \\
\hline 7 & 7 & $\mathrm{R} \$ 691,73$ & $\mathrm{R} \$ 7.502,09$ & $\mathrm{R} \$ 1.096,72$ & $\mathrm{R} \$ 9.290,54$ & 2,39 \\
\hline 7 & 7 & $\mathrm{R} \$ 691,73$ & $\mathrm{R} \$ 10.002,78$ & $\mathrm{R} \$ 1.096,72$ & $\mathrm{R} \$ 11.791,23$ & 1,87 \\
\hline 7 & 7 & $\mathrm{R} \$ 691,73$ & $\mathrm{R} \$ 12.503,48$ & $\mathrm{R} \$ 1.096,72$ & $\mathrm{R} \$ 14.291,93$ & 1,54 \\
\hline 10 & 10 & $\mathrm{R} \$ 785,35$ & $\mathrm{R} \$ 3.002,07$ & $\mathrm{R} \$ 709,94$ & $\mathrm{R} \$ 4.497,35$ & 3,92 \\
\hline 10 & 10 & $\mathrm{R} \$ 785,35$ & $\mathrm{R} \$ 6.004,13$ & $\mathrm{R} \$ 709,94$ & $\mathrm{R} \$ 7.499,42$ & 1,97 \\
\hline 9 & 9 & $\mathrm{R} \$ 785,35$ & $\mathrm{R} \$ 7.518,40$ & $\mathrm{R} \$ 2.033,06$ & $\mathrm{R} \$ 10.336,80$ & 0,57 \\
\hline 9 & 9 & $\mathrm{R} \$ 785,35$ & $\mathrm{R} \$ 10.024,53$ & $\mathrm{R} \$ 2.033,06$ & $\mathrm{R} \$ 12.842,94$ & 0,58 \\
\hline 9 & 9 & $\mathrm{R} \$ 785,35$ & $\mathrm{R} \$ 12.530,66$ & $\mathrm{R} \$ 2.033,06$ & $\mathrm{R} \$ 15.349,07$ & 0,71 \\
\hline 11 & 11 & $\mathrm{R} \$ 785,35$ & $\mathrm{R} \$ 3.500,65$ & $\mathrm{R} \$ 457,61$ & $\mathrm{R} \$ 4.743,61$ & 2,94 \\
\hline 10 & 10 & $\mathrm{R} \$ 785,35$ & $\mathrm{R} \$ 6.004,13$ & $\mathrm{R} \$ 1.419,88$ & $\mathrm{R} \$ 8.209,36$ & 1,67 \\
\hline 10 & 10 & $\mathrm{R} \$ 785,35$ & $\mathrm{R} \$ 9.006,20$ & $\mathrm{R} \$ 1.419,88$ & $\mathrm{R} \$ 11.211,43$ & 0,78 \\
\hline 10 & 10 & $\mathrm{R} \$ 785,35$ & $\mathrm{R} \$ 12.008,26$ & $\mathrm{R} \$ 1.419,88$ & $\mathrm{R} \$ 14.213,49$ & 0,86 \\
\hline 10 & 10 & $\mathrm{R} \$ 785,35$ & $\mathrm{R} \$ 15.010,33$ & $\mathrm{R} \$ 1.419,88$ & $\mathrm{R} \$ 17.215,56$ & 0,92 \\
\hline 11 & 11 & $\mathrm{R} \$ 785,35$ & $\mathrm{R} \$ 3.500,65$ & $\mathrm{R} \$ 686,42$ & $\mathrm{R} \$ 4.972,42$ & 3,20 \\
\hline 11 & 11 & $\mathrm{R} \$ 785,35$ & $\mathrm{R} \$ 7.001,29$ & $\mathrm{R} \$ 686,42$ & $\mathrm{R} \$ 8.473,06$ & 1,64 \\
\hline 10 & 10 & $\mathrm{R} \$ 785,35$ & $\mathrm{R} \$ 9.006,20$ & $\mathrm{R} \$ 2.129,82$ & $\mathrm{R} \$ 11.921,37$ & 1,01 \\
\hline 10 & 10 & $\mathrm{R} \$ 785,35$ & $\mathrm{R} \$ 12.008,26$ & $\mathrm{R} \$ 2.129,82$ & $\mathrm{R} \$ 14.923,43$ & 0,39 \\
\hline 10 & 10 & $\mathrm{R} \$ 785,35$ & $\mathrm{R} \$ 15.010,33$ & $\mathrm{R} \$ 2.129,82$ & $\mathrm{R} \$ 17.925,50$ & 0,36 \\
\hline 12 & 12 & $\mathrm{R} \$ 785,35$ & $\mathrm{R} \$ 4.000,19$ & $\mathrm{R} \$ 273,72$ & $\mathrm{R} \$ 5.059,25$ & 3,22 \\
\hline 11 & 11 & $\mathrm{R} \$ 785,35$ & $\mathrm{R} \$ 7.001,29$ & $\mathrm{R} \$ 915,23$ & $\mathrm{R} \$ 8.701,87$ & 1,20 \\
\hline 11 & 11 & $\mathrm{R} \$ 785,35$ & $\mathrm{R} \$ 10.501,94$ & $\mathrm{R} \$ 915,23$ & $\mathrm{R} \$ 12.202,51$ & 1,14 \\
\hline 10 & 10 & $\mathrm{R} \$ 785,35$ & $\mathrm{R} \$ 12.008,26$ & $\mathrm{R} \$ 2.839,77$ & $\mathrm{R} \$ 15.633,38$ & 0,66 \\
\hline 10 & 10 & $\mathrm{R} \$ 785,35$ & $\mathrm{R} \$ 15.010,33$ & $\mathrm{R} \$ 2.839,77$ & $\mathrm{R} \$ 18.635,44$ & 0,32 \\
\hline 14 & 14 & $\mathrm{R} \$ 798,02$ & $\mathrm{R} \$ 4.001,08$ & $\mathrm{R} \$ 350,09$ & $\mathrm{R} \$ 5.149,19$ & 1,50 \\
\hline 13 & 13 & $\mathrm{R} \$ 798,02$ & $\mathrm{R} \$ 7.005,79$ & $\mathrm{R} \$ 907,12$ & $\mathrm{R} \$ 8.710,93$ & 0,49 \\
\hline 12 & 12 & $\mathrm{R} \$ 798,02$ & $\mathrm{R} \$ 9.021,93$ & $\mathrm{R} \$ 2.206,87$ & $\mathrm{R} \$ 12.026,82$ & 0,23 \\
\hline 12 & 12 & $\mathrm{R} \$ 798,02$ & $\mathrm{R} \$ 12.029,24$ & $\mathrm{R} \$ 2.206,87$ & $\mathrm{R} \$ 15.034,13$ & 0,23 \\
\hline 12 & 12 & $\mathrm{R} \$ 798,02$ & $\mathrm{R} \$ 15.036,55$ & $\mathrm{R} \$ 2.206,87$ & $\mathrm{R} \$ 18.041,44$ & 0,23 \\
\hline 14 & 14 & $\mathrm{R} \$ 798,02$ & $\mathrm{R} \$ 4.001,08$ & $\mathrm{R} \$ 700,18$ & $\mathrm{R} \$ 5.499,28$ & 0,70 \\
\hline 14 & 14 & $\mathrm{R} \$ 798,02$ & $\mathrm{R} \$ 8.002,17$ & $\mathrm{R} \$ 700,18$ & $\mathrm{R} \$ 9.500,36$ & 0,48 \\
\hline 13 & 13 & $\mathrm{R} \$ 798,02$ & $\mathrm{R} \$ 10.508,69$ & $\mathrm{R} \$ 1.814,25$ & $\mathrm{R} \$ 13.120,96$ & 0,23 \\
\hline 13 & 13 & $\mathrm{R} \$ 798,02$ & $\mathrm{R} \$ 14.011,59$ & $\mathrm{R} \$ 1.814,25$ & $\mathrm{R} \$ 16.623,85$ & 0,23 \\
\hline 13 & 13 & $\mathrm{R} \$ 798,02$ & $\mathrm{R} \$ 17.514,49$ & $\mathrm{R} \$ 1.814,25$ & $\mathrm{R} \$ 20.126,75$ & 0,23 \\
\hline 15 & 15 & $\mathrm{R} \$ 798,02$ & $\mathrm{R} \$ 4.500,38$ & $\mathrm{R} \$ 381,82$ & $\mathrm{R} \$ 5.680,22$ & 0,96 \\
\hline 14 & 14 & $\mathrm{R} \$ 798,02$ & $\mathrm{R} \$ 8.002,17$ & $\mathrm{R} \$ 1.050,27$ & $\mathrm{R} \$ 9.850,45$ & 0,38 \\
\hline 14 & 14 & $\mathrm{R} \$ 798,02$ & $\mathrm{R} \$ 12.003,25$ & $\mathrm{R} \$ 1.050,27$ & $\mathrm{R} \$ 13.851,53$ & 0,32 \\
\hline 13 & 13 & $\mathrm{R} \$ 798,02$ & $\mathrm{R} \$ 14.011,59$ & $\mathrm{R} \$ 2.721,37$ & $\mathrm{R} \$ 17.530,98$ & 0,11 \\
\hline 13 & 13 & $\mathrm{R} \$ 798,02$ & $\mathrm{R} \$ 17.514,49$ & $\mathrm{R} \$ 2.721,37$ & $\mathrm{R} \$ 21.033,87$ & 0,12 \\
\hline 15 & 15 & $\mathrm{R} \$ 798,02$ & $\mathrm{R} \$ 4.500,38$ & $\mathrm{R} \$ 509,10$ & $\mathrm{R} \$ 5.807,50$ & 0,72 \\
\hline 14 & 14 & $\mathrm{R} \$ 798,02$ & $\mathrm{R} \$ 8.002,17$ & $\mathrm{R} \$ 1.400,35$ & $\mathrm{R} \$ 10.200,54$ & 0,28 \\
\hline 14 & 14 & $\mathrm{R} \$ 798,02$ & $\mathrm{R} \$ 12.003,25$ & $\mathrm{R} \$ 1.400,35$ & $\mathrm{R} \$ 14.201,62$ & 0,26 \\
\hline 14 & 14 & $\mathrm{R} \$ 798,02$ & $\mathrm{R} \$ 16.004,33$ & $\mathrm{R} \$ 1.400,35$ & $\mathrm{R} \$ 18.202,70$ & 0,24 \\
\hline 13 & 13 & $\mathrm{R} \$ 798,02$ & $\mathrm{R} \$ 17.514,49$ & $\mathrm{R} \$ 3.628,49$ & $\mathrm{R} \$ 21.941,00$ & 0,05 \\
\hline
\end{tabular}


Tabela 7. Continuação...

\begin{tabular}{|c|c|c|c|c|c|c|c|c|c|c|}
\hline \multicolumn{5}{|c|}{ Parâmetros } & \multicolumn{6}{|c|}{ Solução ótima } \\
\hline $\begin{array}{l}\text { Media da } \\
\text { demanda }\end{array}$ & $\mathbf{M}$ & Caq & CR & i & $\mathbf{S}$ & $\mathbf{s}$ & Ressuprimento & Excesso & Falta & Total \\
\hline 8 & $\mathrm{R} \$ 250.000,00$ & $\mathrm{R} \$ 10.000,00$ & $\$ 800,00$ & $5 \%$ & 17 & 14 & $\mathrm{R} \$ 766,49$ & $\mathrm{R} \$ 4.489,46$ & $\mathrm{R} \$ 417,56$ & $\$ 5.673,52$ \\
\hline 8 & $\mathrm{R} \$ 250.000,00$ & $\mathrm{R} \$ 20.000,00$ & $\mathrm{R} \$ 800,00$ & $5 \%$ & 16 & 13 & $\mathrm{R} \$ 766,49$ & $\mathrm{R} \$ 7.982,80$ & $\mathrm{R} \$ 968,38$ & $\mathrm{R} \$ 9.717,67$ \\
\hline 8 & $\mathrm{R} \$ 250.000,00$ & $\mathrm{R} \$ 30.000,00$ & $\mathrm{R} \$ 800,00$ & $5 \%$ & 15 & 13 & $\mathrm{R} \$ 789,02$ & $\mathrm{R} \$ 10.517,94$ & $\mathrm{R} \$ 2.063,79$ & $\mathrm{R} \$ 13.370,75$ \\
\hline 8 & $\mathrm{R} \$ 250.000,00$ & $\mathrm{R} \$ 40.000,00$ & $\mathrm{R} \$ 800,00$ & $5 \%$ & 15 & 13 & $\mathrm{R} \$ 789,02$ & $\mathrm{R} \$ 14.023,92$ & $\mathrm{R} \$ 2.063,79$ & $\mathrm{R} \$ 16.876,73$ \\
\hline 8 & $\mathrm{R} \$ 250.000,00$ & $\mathrm{R} \$ 50.000,00$ & $\$ 800,00$ & $5 \%$ & 14 & 12 & $\mathrm{R} \$ 789,02$ & $\mathrm{R} \$ 15.073,15$ & $\mathrm{R} \$ 4.325,58$ & $\mathrm{R} \$ 20.187,75$ \\
\hline 8 & $\mathrm{R} \$ 500.000,00$ & $\mathrm{R} \$ 10.000,00$ & $\$ 800,00$ & $5 \%$ & 17 & 15 & $\mathrm{R} \$ 7$ & $\mathrm{R} \$ 4.499,99$ & $\mathrm{R} \$ 799,97$ & $\mathrm{R} \$ 6.088,98$ \\
\hline 8 & $\mathrm{R} \$ 500.000,00$ & $\mathrm{R} \$ 20.000,00$ & $\$ 800,00$ & $5 \%$ & 17 & 14 & $\mathrm{R} \$ 766,49$ & $\mathrm{R} \$ 8.978,92$ & $\mathrm{R} \$ 835,13$ & $\mathrm{R} \$ 10.580,54$ \\
\hline 8 & $\mathrm{R} \$ 500.000,00$ & $\mathrm{R} \$ 30.000,00$ & $\mathrm{R} \$ 800,00$ & $5 \%$ & 16 & 14 & $\mathrm{R} \$ 789,02$ & $\mathrm{R} \$ 12.005,56$ & $\mathrm{R} \$ 1.865,05$ & $\mathrm{R} \$ 14.659,63$ \\
\hline 8 & $\mathrm{R} \$ 500.000,00$ & $\mathrm{R} \$ 40.000,00$ & $\mathrm{R} \$ 800,00$ & $5 \%$ & 16 & 14 & 9,02 & $\mathrm{R} \$ 16.007,41$ & $\mathrm{R} \$ 1.865,05$ & $\mathrm{R} \$ 18.661,48$ \\
\hline 8 & $\mathrm{R} \$ 500.000,00$ & $\mathrm{R} \$ 50.000,00$ & $\mathrm{R} \$ 800,00$ & $5 \%$ & 15 & 13 &, 02 & $\mathrm{R} \$ 17$ & $\mathrm{R} \$ 4.127,59$ & $\mathrm{R} \$ 22.446,51$ \\
\hline 8 & $\mathrm{R} \$ 750.000,00$ & $\mathrm{R} \$ 10.000,00$ & $\mathrm{R} \$ 800,00$ & $5 \%$ & 18 & 15 &, 49 & $\mathrm{R} \$ 4.9$ & $\mathrm{R} \$ 514,05$ & $\mathrm{R} \$ 6.269,16$ \\
\hline 8 & $\mathrm{R} \$ 750.000,00$ & $\mathrm{R} \$ 20.000,00$ & $\$ 800,00$ & $5 \%$ & 17 & 15 &, 02 & $\mathrm{R} \$ 8$. & $\mathrm{R} \$ 1.199,96$ & $\mathrm{R} \$ 10.988,95$ \\
\hline 8 & $\mathrm{R} \$ 750.000,00$ & $\mathrm{R} \$ 30.000,00$ & $\$ 800,00$ & $5 \%$ & 17 & 14 & 49 & $\mathrm{R} \$ 13$. & $\mathrm{R} \$ 1.2$ & $\mathrm{R} \$ 15$. \\
\hline 8 & $\mathrm{R} \$ 750.000,00$ & $\mathrm{R} \$ 40.000,00$ & $\mathrm{R} \$ 800,00$ & $5 \%$ & 16 & 14 &, 02 & $\mathrm{R} \$ 16.007,41$ & $\mathrm{R} \$ 2.797,58$ & $\mathrm{R} \$ 19.594,01$ \\
\hline 8 & $\mathrm{R} \$ 750.000,00$ & $\mathrm{R} \$ 50.000,00$ & $\mathrm{R} \$ 800,00$ & $5 \%$ & 16 & 14 &, 02 & $\mathrm{R} \$ 20.009,26$ & $\mathrm{R} \$ 2.797,58$ & $\mathrm{R} \$ 23.595,86$ \\
\hline 8 & $\mathrm{R} \$ 1.000 .000,00$ & $\mathrm{R} \$ 10.000,00$ & $\mathrm{R} \$ 800,00$ & $5 \%$ & 18 & 15 & 49 & $\mathrm{R} \$ 4.988,63$ & $\mathrm{R} \$ 685,40$ & $\mathrm{R} \$ 6.4$ \\
\hline 8 & $\mathrm{R} \$ 1.000 .000,00$ & $\mathrm{R} \$ 20.000,00$ & $\mathrm{R} \$ 800,00$ & $5 \%$ & 17 & 15 & 02 & $\mathrm{R} \$ 8$ & $\mathrm{R} \$ 1$ & $\mathrm{R} \$ 11$. \\
\hline 8 & $\mathrm{R} \$ 1.000 .000,00$ & $\mathrm{R} \$ 30.000,00$ & $\mathrm{R} \$ 800,00$ & $5 \%$ & 17 & 15 & $\mathrm{R} \$$ & $\mathrm{R} \$ 1$ & $\mathrm{R} \$ 1$ & $\mathrm{R} \$ 1$ \\
\hline 8 & $\mathrm{R} \$ 1.000 .000,00$ & $\mathrm{R} \$ 40.000,00$ & $\mathrm{R} \$ 800,00$ & $5 \%$ & 17 & 15 & 02 & $\mathrm{R} \$ 17$ & $\mathrm{R} \$ 1$ & $\mathrm{R} \$ 20$ \\
\hline 8 & $\mathrm{R} \$ 1.000 .000,00$ & $\mathrm{R} \$ 50.000,00$ & $\mathrm{R} \$ 800,00$ & $5 \%$ & 16 & 14 & $\mathrm{R} \$$ & $\mathrm{R} \$ 20$ & $\mathrm{R} \$ 3$ & $\mathrm{R} \$ 24$ \\
\hline 10 & $\mathrm{R} \$ 250.000,00$ & $\mathrm{R} \$ 10.000,00$ & $\mathrm{R} \$ 800,00$ & $5 \%$ & 19 & 16 & 1,75 & $\mathrm{R} \$ 4.500,65$ & $\mathrm{R} \$ 870,13$ & $\mathrm{R} \$ 6.162,54$ \\
\hline 10 & $\mathrm{R} \$ 250.000,00$ & $\mathrm{R} \$ 20.000,00$ & $\mathrm{R} \$ 800,00$ & $5 \%$ & 18 & 16 & $\mathrm{R} \$ 797,79$ & $\mathrm{R} \$ 8.012,97$ & $\mathrm{R} \$ 1.797,43$ & $\mathrm{R} \$ 10.608,19$ \\
\hline 10 & $\mathrm{R} \$ 250.000,00$ & $\mathrm{R} \$ 30.000,00$ & $\mathrm{R} \$ 800,00$ & $5 \%$ & 18 & 15 & $\mathrm{R} \$ 791,75$ & $\mathrm{R} \$ 12.012,81$ & $\mathrm{R} \$ 1.808,67$ & $\mathrm{R} \$ 14.613,23$ \\
\hline 10 & $\mathrm{R} \$ 250.000,00$ & $\mathrm{R} \$ 40.000,00$ & $\mathrm{R} \$ 800,00$ & $5 \%$ & 17 & 15 & $\mathrm{R} \$ 7$ & $\mathrm{R} \$ 14$ & $\mathrm{R} \$ 3$. & $\mathrm{R} \$ 18.4$ \\
\hline 10 & $\mathrm{R} \$ 250.000,00$ & $\mathrm{R} \$ 50.000,00$ & & $5 \%$ & 17 & 15 & $\mathrm{R} \$ 797$ & $\mathrm{R} \$ 17$ & $\mathrm{R} \$ ?$ & $\mathrm{R} \$ 21.9$ \\
\hline 10 & $\mathrm{R} \$ 500.000,00$ & $\mathrm{R} \$ 10.000,00$ & $\mathrm{R} \$ 800,00$ & $5 \%$ & 20 & 17 & $\mathrm{R} \$ 7$ & $\mathrm{R} \$ 4.998,91$ & $\mathrm{R} \$ 800,89$ & $\mathrm{R} \$ 6.591,55$ \\
\hline 10 & $\mathrm{R} \$ 500.000,00$ & $\mathrm{R} \$ 20.000,00$ & $\mathrm{R} \$ 800,00$ & $5 \%$ & 19 & 17 & $\mathrm{R} \$ 797,79$ & $\mathrm{R} \$ 9.005,78$ & $\mathrm{R} \$ 1.728,02$ & $\mathrm{R} \$ 11.531,59$ \\
\hline 10 & $\mathrm{R} \$ 500.000,00$ & $\mathrm{R} \$ 30.000,00$ & $\mathrm{R} \$ 800,00$ & $5 \%$ & 19 & 16 & $\mathrm{R} \$ 791,7$ & $\mathrm{R} \$ 13.501,96$ & $\mathrm{R} \$ 1.740,27$ & $\mathrm{R} \$ 16.033,98$ \\
\hline 10 & $\mathrm{R} \$ 500.000,00$ & $\mathrm{R} \$ 40.000,00$ & $\mathrm{R} \$ 800,00$ & $5 \%$ & 18 & 16 & $\mathrm{R} \$ 797,79$ & $\mathrm{R} \$ 16.025,94$ & $\mathrm{R} \$ 3.594,86$ & $\mathrm{R} \$ 20.418,59$ \\
\hline 10 & $\mathrm{R} \$ 500.000,00$ & $\mathrm{R} \$ 50.000,00$ & $\mathrm{R} \$ 800,00$ & $5 \%$ & 18 & 16 & $\mathrm{R} \$ 797,79$ & $\mathrm{R} \$ 20.032,43$ & $\mathrm{R} \$ 3.594,86$ & $\mathrm{R} \$ 24.425,08$ \\
\hline 10 & $\mathrm{R} \$ 750.000,00$ & $\mathrm{R} \$ 10.000,00$ & $\mathrm{R} \$ 800,00$ & $5 \%$ & 21 & 18 & $\mathrm{R} \$ 791,75$ & $\mathrm{R} \$ 5.498,11$ & $\mathrm{R} \$ 529,72$ & $\mathrm{R} \$ 6.819,58$ \\
\hline 10 & $\mathrm{R} \$ 750.000,00$ & $\mathrm{R} \$ 20.000,00$ & $\mathrm{R} \$ 800,00$ & $5 \%$ & 20 & 17 & $\mathrm{R} \$ 791,75$ & $\mathrm{R} \$ 9.997,82$ & $\mathrm{R} \$ 1.201,34$ & $\mathrm{R} \$ 11.990,91$ \\
\hline 10 & $\mathrm{R} \$ 750.000,00$ & $\mathrm{R} \$ 30.000,00$ & $\mathrm{R} \$ 800,00$ & $5 \%$ & 19 & 17 & $\mathrm{R} \$ 797,79$ & $\mathrm{R} \$ 13.508,67$ & $\mathrm{R} \$ 2.592,03$ & $\mathrm{R} \$ 16.898,49$ \\
\hline 10 & $\mathrm{R} \$ 750.000,00$ & $\mathrm{R} \$ 40.000,00$ & $\mathrm{R} \$ 800,00$ & $5 \%$ & 19 & 17 & $\mathrm{R} \$ 797,79$ & $\mathrm{R} \$ 18.011,56$ & $\mathrm{R} \$ 2.592,03$ & $\mathrm{R} \$ 21.401,38$ \\
\hline 10 & $\mathrm{R} \$ 750.000,00$ & $\mathrm{R} \$ 50.000,00$ & $\mathrm{R} \$ 800,00$ & $5 \%$ & 19 & 17 & $\mathrm{R} \$ 797,79$ & $\mathrm{R} \$ 22.514,45$ & $\mathrm{R} \$ 2.592,03$ & $\mathrm{R} \$ 25.904,27$ \\
\hline 10 & $\mathrm{R} \$ 1.000 .000,00$ & $\mathrm{R} \$ 10.000,00$ & $\mathrm{R} \$ 800,00$ & $5 \%$ & 21 & 18 & $\mathrm{R} \$ 791,75$ & $\mathrm{R} \$ 5.498,11$ & $\mathrm{R} \$ 706,29$ & $\mathrm{R} \$ 6.996,15$ \\
\hline 10 & $\mathrm{R} \$ 1.000 .000,00$ & $\mathrm{R} \$ 20.000,00$ & $\mathrm{R} \$ 800,00$ & $5 \%$ & 20 & 18 & $\mathrm{R} \$ 797,79$ & $\mathrm{R} \$ 10.002,33$ & $\mathrm{R} \$ 1.589,11$ & $\mathrm{R} \$ 12.389,22$ \\
\hline 10 & $\mathrm{R} \$ 1.000 .000,00$ & $\mathrm{R} \$ 30.000,00$ & $\mathrm{R} \$ 800,00$ & $5 \%$ & 20 & 17 & $\mathrm{R} \$ 791,75$ & $\mathrm{R} \$ 14.996,73$ & $\mathrm{R} \$ 1.601,78$ & $\mathrm{R} \$ 17.390,27$ \\
\hline 10 & $\mathrm{R} \$ 1.000 .000,00$ & $\mathrm{R} \$ 40.000,00$ & $\mathrm{R} \$ 800,00$ & $5 \%$ & 19 & 17 & $\mathrm{R} \$ 797,79$ & $\mathrm{R} \$ 18.011,56$ & $\mathrm{R} \$ 3.456,04$ & $\mathrm{R} \$ 22.265,38$ \\
\hline 10 & $\mathrm{R} \$ 1.000 .000,00$ & $\mathrm{R} \$ 50.000,00$ & $\mathrm{R} \$ 800,00$ & $5 \%$ & 19 & 17 & $\mathrm{R} \$ 797,79$ & $\mathrm{R} \$ 22.514,45$ & $\mathrm{R} \$ 3.456,04$ & $\mathrm{R} \$ 26.768,28$ \\
\hline
\end{tabular}


Heurística

Variação percentual

\begin{tabular}{|c|c|c|c|c|c|c|}
\hline \multicolumn{6}{|c|}{ Heuristica } & \multirow[t]{2}{*}{ variaçao percentual } \\
\hline $\mathbf{S}^{\prime}$ & $\mathbf{s}^{\prime}$ & Ressuprimento & Excesso & Falta & Total & \\
\hline 17 & 17 & $\mathrm{R} \$ 799,73$ & $\mathrm{R} \$ 4.501,32$ & $\mathrm{R} \$ 398,57$ & $\mathrm{R} \$ 5.699,62$ & 0,46 \\
\hline 16 & 16 & $\mathrm{R} \$ 799,73$ & $\mathrm{R} \$ 8.006,36$ & $\mathrm{R} \$ 929,51$ & $\mathrm{R} \$ 9.735,60$ & 0,18 \\
\hline 15 & 15 & $\mathrm{R} \$ 799,73$ & $\mathrm{R} \$ 10.521,89$ & $\mathrm{R} \$ 2.057,75$ & $\mathrm{R} \$ 13.379,37$ & 0,06 \\
\hline 15 & 15 & $\mathrm{R} \$ 799,73$ & $\mathrm{R} \$ 14.029,18$ & $\mathrm{R} \$ 2.057,75$ & $\mathrm{R} \$ 16.886,67$ & 0,06 \\
\hline 14 & 14 & $\mathrm{R} \$ 799,73$ & $\mathrm{R} \$ 15.079,62$ & $\mathrm{R} \$ 4.314,25$ & $\mathrm{R} \$ 20.193,60$ & 0,03 \\
\hline 17 & 17 & $\mathrm{R} \$ 799,73$ & $\mathrm{R} \$ 4.501,32$ & $\mathrm{R} \$ 797,13$ & $\mathrm{R} \$ 6.098,18$ & 0,15 \\
\hline 17 & 17 & $\mathrm{R} \$ 799,73$ & $\mathrm{R} \$ 9.002,64$ & $\mathrm{R} \$ 797,13$ & $\mathrm{R} \$ 10.599,50$ & 0,18 \\
\hline 16 & 16 & $\mathrm{R} \$ 799,73$ & $\mathrm{R} \$ 12.009,54$ & $\mathrm{R} \$ 1.859,01$ & $\mathrm{R} \$ 14.668,28$ & 0,06 \\
\hline 16 & 16 & $\mathrm{R} \$ 799,73$ & $\mathrm{R} \$ 16.012,72$ & $\mathrm{R} \$ 1.859,01$ & $\mathrm{R} \$ 18.671,46$ & 0,05 \\
\hline 15 & 15 & $\mathrm{R} \$ 799,73$ & $\mathrm{R} \$ 17.536,48$ & $\mathrm{R} \$ 4.115,51$ & $\mathrm{R} \$ 22.451,71$ & 0,02 \\
\hline 18 & 18 & $\mathrm{R} \$ 799,73$ & $\mathrm{R} \$ 5.000,52$ & $\mathrm{R} \$ 487,78$ & $\mathrm{R} \$ 6.288,03$ & 0,30 \\
\hline 17 & 17 & $\mathrm{R} \$ 799,73$ & $\mathrm{R} \$ 9.002,64$ & $\mathrm{R} \$ 1.195,70$ & $\mathrm{R} \$ 10.998,07$ & 0,08 \\
\hline 17 & 17 & $\mathrm{R} \$ 799,73$ & $\mathrm{R} \$ 13.503,96$ & $\mathrm{R} \$ 1.195,70$ & $\mathrm{R} \$ 15.499,39$ & 0,08 \\
\hline 16 & 16 & $\mathrm{R} \$ 799,73$ & $\mathrm{R} \$ 16.012,72$ & $\mathrm{R} \$ 2.788,52$ & $\mathrm{R} \$ 19.600,97$ & 0,04 \\
\hline 16 & 16 & $\mathrm{R} \$ 799,73$ & $\mathrm{R} \$ 20.015,90$ & $\mathrm{R} \$ 2.788,52$ & $\mathrm{R} \$ 23.604,15$ & 0,04 \\
\hline 18 & 18 & $\mathrm{R} \$ 799,73$ & $\mathrm{R} \$ 5.000,52$ & $\mathrm{R} \$ 650,37$ & $\mathrm{R} \$ 6.450,62$ & 0,16 \\
\hline 17 & 17 & $\mathrm{R} \$ 799,73$ & $\mathrm{R} \$ 9.002,64$ & $\mathrm{R} \$ 1.594,26$ & $\mathrm{R} \$ 11.396,63$ & 0,07 \\
\hline 17 & 17 & $\mathrm{R} \$ 799,73$ & $\mathrm{R} \$ 13.503,96$ & $\mathrm{R} \$ 1.594,26$ & $\mathrm{R} \$ 15.897,96$ & 0,06 \\
\hline 17 & 17 & $\mathrm{R} \$ 799,73$ & $\mathrm{R} \$ 18.005,28$ & $\mathrm{R} \$ 1.594,26$ & $\mathrm{R} \$ 20.399,28$ & 0,05 \\
\hline 16 & 16 & $\mathrm{R} \$ 799,73$ & $\mathrm{R} \$ 20.015,90$ & $\mathrm{R} \$ 3.718,02$ & $\mathrm{R} \$ 24.533,65$ & 0,02 \\
\hline 19 & 19 & $\mathrm{R} \$ 799,96$ & $\mathrm{R} \$ 4.503,12$ & $\mathrm{R} \$ 863,59$ & $\mathrm{R} \$ 6.166,67$ & 0,07 \\
\hline 18 & 18 & $\mathrm{R} \$ 799,96$ & $\mathrm{R} \$ 8.013,42$ & $\mathrm{R} \$ 1.796,63$ & $\mathrm{R} \$ 10.610,01$ & 0,02 \\
\hline 18 & 18 & $\mathrm{R} \$ 799,96$ & $\mathrm{R} \$ 12.020,13$ & $\mathrm{R} \$ 1.796,63$ & $\mathrm{R} \$ 14.616,72$ & 0,02 \\
\hline 17 & 17 & $\mathrm{R} \$ 799,96$ & $\mathrm{R} \$ 14.055,39$ & $\mathrm{R} \$ 3.569,40$ & $\mathrm{R} \$ 18.424,76$ & 0,01 \\
\hline 17 & 17 & $\mathrm{R} \$ 799,96$ & $\mathrm{R} \$ 17.569,24$ & $\mathrm{R} \$ 3.569,40$ & $\mathrm{R} \$ 21.938,61$ & 0,01 \\
\hline 20 & 20 & $\mathrm{R} \$ 799,96$ & $\mathrm{R} \$ 5.001,39$ & $\mathrm{R} \$ 794,13$ & $\mathrm{R} \$ 6.595,48$ & 0,06 \\
\hline 19 & 19 & $\mathrm{R} \$ 799,96$ & $\mathrm{R} \$ 9.006,23$ & $\mathrm{R} \$ 1.727,17$ & $\mathrm{R} \$ 11.533,37$ & 0,02 \\
\hline 19 & 19 & $\mathrm{R} \$ 799,96$ & $\mathrm{R} \$ 13.509,35$ & $\mathrm{R} \$ 1.727,17$ & $\mathrm{R} \$ 16.036,48$ & 0,02 \\
\hline 18 & 18 & $\mathrm{R} \$ 799,96$ & $\mathrm{R} \$ 16.026,84$ & $\mathrm{R} \$ 3.593,25$ & $\mathrm{R} \$ 20.420,05$ & 0,01 \\
\hline 18 & 18 & $\mathrm{R} \$ 799,96$ & $\mathrm{R} \$ 20.033,55$ & $\mathrm{R} \$ 3.593,25$ & $\mathrm{R} \$ 24.426,76$ & 0,01 \\
\hline 21 & 21 & $\mathrm{R} \$ 799,96$ & $\mathrm{R} \$ 5.500,59$ & $\mathrm{R} \$ 524,74$ & $\mathrm{R} \$ 6.825,30$ & 0,08 \\
\hline 20 & 20 & $\mathrm{R} \$ 799,96$ & $\mathrm{R} \$ 10.002,78$ & $\mathrm{R} \$ 1.191,20$ & $\mathrm{R} \$ 11.993,94$ & 0,03 \\
\hline 19 & 19 & $\mathrm{R} \$ 799,96$ & $\mathrm{R} \$ 13.509,35$ & $\mathrm{R} \$ 2.590,76$ & $\mathrm{R} \$ 16.900,07$ & 0,01 \\
\hline 19 & 19 & $\mathrm{R} \$ 799,96$ & $\mathrm{R} \$ 18.012,47$ & $\mathrm{R} \$ 2.590,76$ & $\mathrm{R} \$ 21.403,19$ & 0,01 \\
\hline 19 & 19 & $\mathrm{R} \$ 799,96$ & $\mathrm{R} \$ 22.515,58$ & $\mathrm{R} \$ 2.590,76$ & $\mathrm{R} \$ 25.906,30$ & 0,01 \\
\hline 21 & 21 & $\mathrm{R} \$ 799,96$ & $\mathrm{R} \$ 5.500,59$ & $\mathrm{R} \$ 699,65$ & $\mathrm{R} \$ 7.000,21$ & 0,06 \\
\hline 20 & 20 & $\mathrm{R} \$ 799,96$ & $\mathrm{R} \$ 10.002,78$ & $\mathrm{R} \$ 1.588,26$ & $\mathrm{R} \$ 12.391,00$ & 0,01 \\
\hline 20 & 20 & $\mathrm{R} \$ 799,96$ & $\mathrm{R} \$ 15.004,17$ & $\mathrm{R} \$ 1.588,26$ & $\mathrm{R} \$ 17.392,39$ & 0,01 \\
\hline 19 & 19 & $\mathrm{R} \$ 799,96$ & $\mathrm{R} \$ 18.012,47$ & $\mathrm{R} \$ 3.454,34$ & $\mathrm{R} \$ 22.266,77$ & 0,01 \\
\hline 19 & 19 & $\mathrm{R} \$ 799,96$ & $\mathrm{R} \$ 22.515,58$ & $\mathrm{R} \$ 3.454,34$ & $\mathrm{R} \$ 26.769,89$ & 0,01 \\
\hline
\end{tabular}




\title{
A model for spare parts stock management using Markov chains
}

\begin{abstract}
In this study, we propose a model for a management stock system of spare parts using Markov chains. We compare this method with a conventional simulation showing that both methods are equivalent. In addition, we propose heuristics to find the system parameters based on the properties of Markov Chains and graphics related to the costs implied in the stock management of spare parts.
\end{abstract}

Keywords: Spare parts. Inventory management. Markov chains.

\section{Referências bibliográficas}

BOTTER, R.; FORTUIN, L. Stocking strategy for service parts: a case study. International Journal of Operations \& Production Management, Bingley, UK, v. 20, n. 6, p. 656-674, 2000.

DEKKER, R.; KLEIJN, M.J.; DE ROOIJ, P.J. A spare parts stocking policy based on equipment criticality. International Journal of Production Economics, Oxford, UK, v. 56/57, n. 1-3, p. 69-77, 1998.

FIGUEIREDO, K.F.; FLEURY, P.F.; WANKE, P. Logística e gerenciamento da cadeia de suprimentos. São Paulo: Editora Atlas, 2003. $483 \mathrm{p}$.

GAMERMAN, D. Simulação estocástica via cadeias de Markov. Associação Brasileira de Estatística, 1996. São Paulo: Associação Brasileira de Estatística. p.196.

HILL, R.M.; OMAR, M.; SMITH, D.K. Stock replenishment policies for a stochastic exponentially-declining demand process. European Journal of Operational Research, Oxford, UK, v. 116, p. 374-388, 1999.

HILLIER, F. S.; LIEBERMAN, G. J. Introdução à pesquisa operacional. Rio de Janeiro: Editora Campus, 1988. p.852.

KIM, J.S.; SHIN, K.C.; YU, H.K. Optimal algorithm to determine the spare inventory level for a repairable-item inventory system. Computers \& Operations Research, Oxford, UK, v. 23, n. 3, p. 289-297, 1996.

SANDVIG, J. C.; ALLAIRE, J. J. Vitalizing a service parts inventory. Production and Inventory Management Journal, Alexandria, US, v. 39, n. 1, p. 67-71, 1998.

SHERBROOKE, C.C. Metric: a multi-echelon technique for recoverable item control. Operations Research, Hanover, US, v. 16, p. 122-141, 1968.

SHIBUYA, T.; DOHI, T.; OSAKI, S. Optimal continuous review policies for spare part provisioning with random lead-times. International Journal of Production Economics, Oxford, UK, v. 55, n. 3, p. 257-271, 1998.
SILVER, E; PETERSON, R. Decision systems for inventory management and production planning. New York: Wiley, 1985. 722 p.

SILVER, E.; PYKE, D.; PETERSON, R. Inventory management and production planning and scheduling. New York: Wiley, $1998.754 \mathrm{p}$.

SLEPTCHENKO, A.; HEIJDEN, M.C.; HARTEN, A. Using repair priorities to reduce stock investment in spare part networks. European Journal of Operational Research, Oxford, UK, v. 163, p.733-750, 2005.

SMIDT-DESTOMBES, K.; HEIJDEN, M. C.; HARTEN, A. On the interaction between maintenance, spare part inventories and repair capacity for a k-out-of-N system with wear-out. European Journal of Operational Research, Oxford, UK, v. 174, p. 182-200, 2006.

VAUGHAN, T. S. Failure replacement and preventive maintenance spare parts ordering policy. European Journal of Operational Research, Oxford, UK, v. 161, p.183-190, 2005.

WALKER, J. Base stock level determination of insurance type spares. International Journal of Quality \& Reliability Management, Bingley, UK, v. 14, n. 6, p. 569-574, 1997.

WANKE, P. Gestão de estoques na cadeia de suprimento. São Paulo: Editora Atlas, 2003. 176 p.

WANKE, P. Considerações Metodológicas sobre a Gestão de Estoques de Peças de Reposição: um estudo de caso. In: GUTIERREZ, M.; BERTAND, H. (Org.). Estudos em negócios IV. Rio de Janeiro: Editora Mauad, 2005. (340).

WONG, H.; CATTRYSSE, D.; OUDHEUSDENS, D. Inventory pooling of repairable spare parts with non-zero lateral transshipment time and delayed lateral transshipments. European Journal of Operational Research, Oxford, UK, v. 165, p. 207-218, 2005.

ZIPKIN, P.H. Foundations of inventory management. New York: Irwin, 2000. 514 p.

\section{Sobre os autores}

\section{Antonio Vinicius Pimpão Gomes \\ Peter Wanke}

Centro de Estudos em Logística, Instituto COPPEAD de Administração, Universidade Federal do Rio de Janeiro - UFRJ, Cidade Universitária, Ilha do Fundão, CEP 21949-900, Rio de Janeiro, RJ, Brasil,

e-mails: antonio.pgomes@ coppead.ufrj.br; peter@ coppead.ufrj.br 